
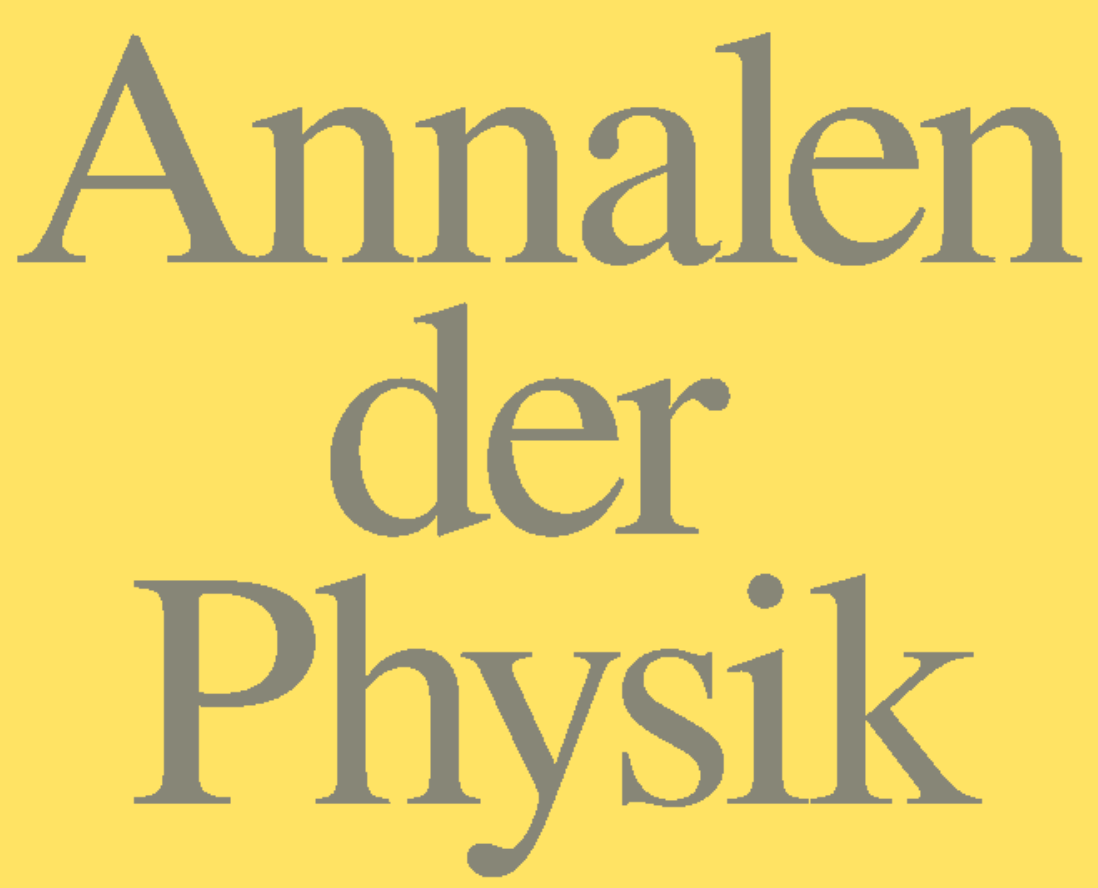

\title{
Solutions of the Häussler-von der Malsburg equations in manifolds with constant curvatures
}

\section{Güßmann ${ }^{1}$, A. Pelster ${ }^{2}$, and G. Wunner ${ }^{1}$}

\footnotetext{
${ }^{1}$ 1. Institut für Theoretische Physik, Universität Stuttgart, Pfaffenwaldring 57, 70569 Stuttgart, Germany

${ }^{2}$ Fachbereich Physik, Campus Duisburg, Universität Duisburg-Essen, Lotharstrasse 1, 47048 Duisburg, Germany
}

Received 12 December 2006, revised 9 February 2007, accepted 26 April 2007 by U. Eckern Published online 11 June 2007

Key words Synergetics, nonlinear dynamics, retinotopy.

PACS 05.45.-a, 87.18.Hf, 89.75.Fb

Dedicated to Hermann Haken on the occasion of his 80th birthday.

We apply generic order parameter equations for the emergence of retinotopy between manifolds of different geometry to one- and two-dimensional Euclidean and spherical manifolds. To this end we elaborate both a linear and a nonlinear synergetic analysis which results in order parameter equations for the dynamics of connection weights between two cell sheets. Our results for strings are analogous to those for discrete linear chains obtained previously by Häussler and von der Malsburg. The case of planes turns out to be more involved as the two dimensions do not decouple in a trivial way. However, superimposing two modes under suitable conditions provides a state with a pronounced retinotopic character. In the case of spherical manifolds we show that the order parameter equations provide stable stationary solutions which correspond to retinotopic modes. A further analysis of higher modes furnishes proof that our model describes the emergence of a perfect one-to-one retinotopy between two spheres.

Ann. Phys. (Leipzig) 16, No. 5 -6, 395 -424 (2007) / DOI 10.1002/andp.200610244 (WI) WILY-VCH 


\title{
Solutions of the Häussler-von der Malsburg equations in manifolds with constant curvatures
}

\author{
M. Güßmann ${ }^{1, *}$, A. Pelster ${ }^{2, * *}$, and G. Wunner ${ }^{1, * * *}$ \\ ${ }^{1}$ 1. Institut für Theoretische Physik, Universität Stuttgart, Pfaffenwaldring 57, 70569 Stuttgart, Germany \\ ${ }^{2}$ Fachbereich Physik, Campus Duisburg, Universität Duisburg-Essen, Lotharstrasse 1, 47048 Duisburg, \\ Germany
}

Received 12 December 2006, revised 9 February 2007, accepted 26 April 2007 by U. Eckern

Published online 11 June 2007

Key words Synergetics, nonlinear dynamics, retinotopy.

PACS 05.45.-a, 87.18.Hf, 89.75.Fb

Dedicated to Hermann Haken on the occasion of his 80th birthday.

\begin{abstract}
We apply generic order parameter equations for the emergence of retinotopy between manifolds of different geometry to one- and two-dimensional Euclidean and spherical manifolds. To this end we elaborate both a linear and a nonlinear synergetic analysis which results in order parameter equations for the dynamics of connection weights between two cell sheets. Our results for strings are analogous to those for discrete linear chains obtained previously by Häussler and von der Malsburg. The case of planes turns out to be more involved as the two dimensions do not decouple in a trivial way. However, superimposing two modes under suitable conditions provides a state with a pronounced retinotopic character. In the case of spherical manifolds we show that the order parameter equations provide stable stationary solutions which correspond to retinotopic modes. A further analysis of higher modes furnishes proof that our model describes the emergence of a perfect one-to-one retinotopy between two spheres.
\end{abstract}

(c) 2007 WILEY-VCH Verlag GmbH \& Co. KGaA, Weinheim

\section{Introduction}

In a preceding paper [1] we have analyzed a general model for the formation of retinotopic projections which is independent of geometry and dimension. In this paper we present applications of the general model, viz Euclidean and spherical geometries in one and two dimensions. To put these investigations into perspective we briefly recall their physiological motivations. But let us stress at the outset that our primary objective is not the biological modelling of retinotopic projections but the systematic analysis of a particular model thereof from a nonlinear dynamics point of view. In the course of ontogenesis of vertebrate animals well-ordered neural connections are established between retina and tectum, a part of the brain which plays an important role in processing optical information. At an initial stage of ontogenesis, the ganglion cells of the retina have random synaptic contacts with the tectum. In the adult animal, however, neighbouring retinal cells project onto neighbouring cells of the tectum [2]. A detailed analytical treatment by Häussler and von der Malsburg was able to describe the generation of such retinotopic states from an undifferentiated initial state as a self-organization process [3]. In that work retina and tectum were treated as one-dimensional discrete cell arrays. The dynamics of the connection weights between retina and tectum was assumed to be governed by the Häussler-von der Malsburg equations which are based on modelling the interplay between cooperative and competitive interactions of the individual synaptic contacts. The nonlinear analysis was

* E-mail: martin.guessmann@itp1.uni-stuttgart.de

** Corresponding author E-mail: axel.pelster@uni-due.de

*** E-mail: guenter.wunner@itp1.uni-stuttgart.de 


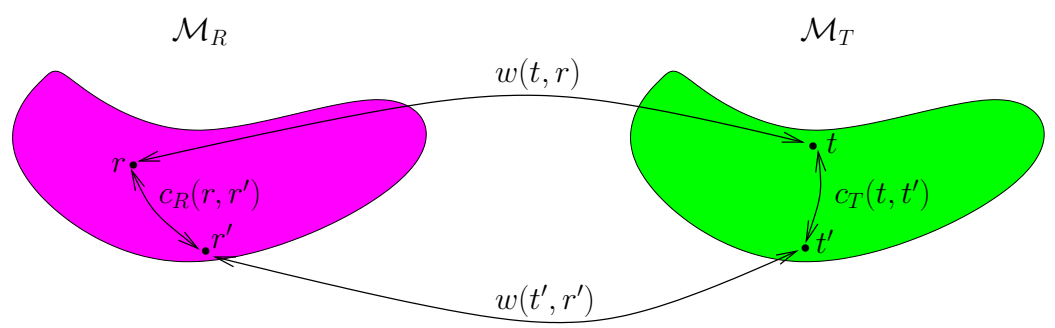

Fig. 1 (online colour at: www.ann-phys.org) Retina and tectum are represented as manifolds $\mathcal{M}_{R}$ and $\mathcal{M}_{T}$, respectively, which are connected by positive connection weights $w(t, r)$. The connectivity within each manifold is represented by cooperativity functions $c_{R}\left(r, r^{\prime}\right)$ and $c_{T}\left(t, t^{\prime}\right)$.

performed using the methods of synergetics, which provides effective analytical methods to study selforganization processes in complex systems $[4,5]$.

Obviously, the description of cell sheets as linear chains with the same number of cells is an inadequate approach to the real biological situation. In a preceding paper we generalized the underlying Häussler-von der Malsburg equations to continuous manifolds of arbitrary geometry and dimension [1]. We performed an extensive synergetic analysis of these generalized Häussler-von der Malsburg equations. The resulting generic order parameter equations represented a central new result, and can now serve as a starting point to analyze in detail the self-organized emergence of one-to-one mappings in cell arrays of different geometries. A short review of our generalization of the Häussler-von der Malsburg equations and the results of the corresponding synergetic analysis is provided in Sect. 2. In the subsequent two Sections we focus on oneand two-dimensional Euclidean manifolds. We show in Sect. 3 that the treatment of strings yields results which are analogous to those obtained for discrete linear chains in [3], i.e. our model includes the special case discussed by Häussler and von der Malsburg. However, our synergetic analysis is more general. Instead of discrete cell arrays with the same number of cells, we consider continuously distributed cells on strings of different lengths [6]. Furthermore, we do not restrict our investigations to monotonically decreasing cooperativity functions of strings. We investigate under what circumstances non-retinotopic modes become unstable and destroy retinotopic order. We show in Sect. 4 that our generic order parameter equations also provide a suitable framework to describe the emergence of retinotopy between planes. For a certain superposition of two modes we demonstrate that taking into account the contribution of the higher modes leads to a sharpening of the retinotopic character of the projection between the two cell sheets. Finally, we analyse in Sect. 5 the formation of retinotopic projections for the biologically relevant situation of spherical geometries, i.e. manifolds with positive constant curvature. It turns out that the case of spheres exhibits remarkable similarities with the analysis of strings, especially regarding the generation of 1-1retinotopic projections.

\section{The general model}

To make the present paper self-contained, we briefly review the essential results of our general model for the self-organized emergence of retinotopic projections between manifolds of different geometry in [1]. The two cell sheets, retina and tectum, are represented by general manifolds $\mathcal{M}_{R}$ and $\mathcal{M}_{T}$, respectively. Every ordered pair $(t, r)$ with $t \in \mathcal{M}_{T}, r \in \mathcal{M}_{R}$ is connected by a connection weight $w(t, r)$ as is illustrated in Fig. 1. The equations of evolution of these connection weights are assumed to be given by a generalization of the Häussler-von der Malsburg equations

$$
\dot{w}(t, r)=f(t, r, w)-\frac{w(t, r)}{2 M_{T}} \int d t^{\prime} f\left(t^{\prime}, r, w\right)-\frac{w(t, r)}{2 M_{R}} \int d r^{\prime} f\left(t, r^{\prime}, w\right),
$$


where the first term on the right-hand side describes cooperative synaptic growth processes and the other terms stand for corresponding competitive growth processes. Here $M_{T}, M_{R}$ denote the magnitudes of the manifolds, the total growth rates are defined by

$$
f(t, r, w)=\alpha+w(t, r) \int d t^{\prime} \int d r^{\prime} c_{T}\left(t, t^{\prime}\right) c_{R}\left(r, r^{\prime}\right) w\left(t^{\prime}, r^{\prime}\right),
$$

and $\alpha$ is the global growth rate of new synapses onto the tectum which represents the control parameter of our system. The cooperativity functions $c_{T}\left(t, t^{\prime}\right), c_{R}\left(r, r^{\prime}\right)$ represent the neural connectivity within each manifold. We assume that they are positive, symmetric with respect to their arguments and normalized. The cooperation strength depends on the distance between two points of the manifold. This requires a measure of distance, i.e. metrics, which in turn define Laplace-Beltrami operators on the manifolds. Their eigenvalue problems yield a complete orthonormal system $\psi_{\lambda_{T}}(t), \psi_{\lambda_{R}}(r)$, and the generalized Häussler-von der Malsburg equations are most conveniently transformed to this new basis. For example, the cooperativity functions are expanded in terms of these functions as follows:

$$
c_{T}\left(t, t^{\prime}\right)=\sum_{\lambda_{T}} f_{\lambda_{T}} \psi_{\lambda_{T}}(t) \psi_{\lambda_{T}}^{*}\left(t^{\prime}\right), \quad c_{R}\left(r, r^{\prime}\right)=\sum_{\lambda_{R}} f_{\lambda_{R}} \psi_{\lambda_{R}}(r) \psi_{\lambda_{R}}^{*}\left(r^{\prime}\right) .
$$

The initial state of ontogenesis with randomly distributed synaptic contacts is described by the stationary uniform solution of the generalized Häussler-von der Malsburg equations $w_{0}(t, r)=1$. Its stability is analyzed by linearizing the Häussler-von der Malsburg equations (1) with respect to the deviation $v(t, r)=$ $w(t, r)-w_{0}(t, r)$. The resulting linearized equations read $\dot{v}(t, r)=\hat{L}(t, r, v)$ with the linear operator

$$
\begin{aligned}
\hat{L}(t, r, v)= & -\alpha v(t, r)+\int d t^{\prime} \int d r^{\prime} c_{T}\left(t, t^{\prime}\right) c_{R}\left(r, r^{\prime}\right) v\left(t^{\prime}, r^{\prime}\right) \\
& -\frac{1}{2 M_{T}} \int d t^{\prime}\left[v\left(t^{\prime}, r\right)+\int d t^{\prime \prime} \int d r^{\prime \prime} c_{T}\left(t^{\prime}, t^{\prime \prime}\right) c_{R}\left(r, r^{\prime \prime}\right) v\left(t^{\prime \prime}, r^{\prime \prime}\right)\right] \\
& -\frac{1}{2 M_{R}} \int d r^{\prime}\left[v\left(t, r^{\prime}\right)+\int d t^{\prime \prime} \int d r^{\prime \prime} c_{T}\left(t, t^{\prime \prime}\right) c_{R}\left(r^{\prime}, r^{\prime \prime}\right) v\left(t^{\prime \prime}, r^{\prime \prime}\right)\right] .
\end{aligned}
$$

The eigenvalue problem of the linear operator (4) is solved by the eigenfunctions

$$
v_{\lambda_{T} \lambda_{R}}(t, r)=\psi_{\lambda_{T}}(t) \psi_{\lambda_{R}}(r)
$$

and the following spectrum of eigenvalues:

$$
\Lambda_{\lambda_{T} \lambda_{R}}=\left\{\begin{array}{cc}
-\alpha-1 & \lambda_{T}=\lambda_{R}=0 \\
-\alpha+\frac{1}{2}\left(f_{\lambda_{T}}^{T} f_{\lambda_{R}}^{R}-1\right) & \lambda_{T}=0, \lambda_{R} \neq 0 ; \lambda_{R}=0, \lambda_{T} \neq 0 \\
-\alpha+f_{\lambda_{T}}^{T} f_{\lambda_{R}}^{R} & \text { otherwise. }
\end{array}\right.
$$

The eigenvalue with the largest real part is given by $\Lambda_{\max }=-\alpha+f_{\lambda_{T}^{u}}^{T} f_{\lambda_{R}^{u}}^{R}$, where $\lambda_{T}^{u}, \lambda_{R}^{u}$ denote all those eigenvalues which could become unstable simultaneously. Thus, the instability takes place when the global growth rate reaches its critical value $\alpha_{c}=\operatorname{Re}\left(f_{\lambda_{T}^{u}}^{T} f_{\lambda_{R}^{u}}^{R}\right)$.

The linear stability analysis motivates to treat the nonlinear Häussler-von der Malsburg equations (1) near the instability by decomposing the deviation $v(t, r)=w(t, r)-w_{0}(t, r)$ in unstable and stable contributions according to $v(t, r)=U(t, r)+S(t, r)$. With Einstein's sum convention we have for the unstable modes

$$
U(t, r)=U_{\lambda_{T}^{u} \lambda_{R}^{u}} \psi_{\lambda_{T}^{u}}(t) \psi_{\lambda_{R}^{u}}(r),
$$


and, correspondingly,

$$
S(t, r)=S_{\lambda_{T} \lambda_{R}} \psi_{\lambda_{T}}(t) \psi_{\lambda_{R}}(r)
$$

represents the contribution of the stable modes. Note that the summation in (8) is performed over all parameters $\left(\lambda_{T} ; \lambda_{R}\right)$ except for $\left(\lambda_{T}^{u} ; \lambda_{R}^{u}\right)$, i.e. from now on the parameters $\left(\lambda_{T} ; \lambda_{R}\right)$ stand for the stable modes alone. With the help of the slaving principle of synergetics the original high-dimensional system can be reduced to a low-dimensional one which only contains the unstable amplitudes. The general form of the resulting order parameter equations is independent of the geometry of the problem and reads

$$
\begin{aligned}
& \dot{U}_{\lambda_{T}^{u} \lambda_{R}^{u}}=\Lambda_{\lambda_{T}^{u} \lambda_{R}^{u}} U_{\lambda_{T}^{u} \lambda_{R}^{u}}+A_{\lambda_{R}^{u}, \lambda_{R}^{u}{ }^{\prime} \lambda_{R}^{u \prime \prime}}^{\lambda_{T}^{u}, \lambda^{u \prime} \lambda^{u \prime \prime}} U_{\lambda_{T}^{u \prime} \lambda_{R}^{u}{ }^{\prime}} U_{\lambda_{T}^{u \prime \prime} \lambda_{R}^{u \prime \prime}}
\end{aligned}
$$

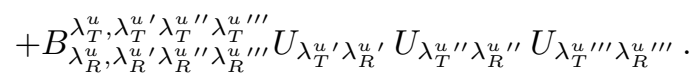

It contains, as is typical, a linear, a quadratic, and a cubic term of the order parameters. The corresponding coefficients can be expressed in terms of the expansion coefficients $f_{\lambda_{T}}, f_{\lambda_{R}}$ of the cooperativity functions (3) and integrals over products of the eigenfunctions $\psi_{\lambda_{T}}(t), \psi_{\lambda_{R}}(r)$ :

$$
\begin{aligned}
I_{\lambda^{(1)} \lambda^{(2)} \ldots \lambda^{(n)}}^{\lambda} & =\int d x \psi_{\lambda}^{*}(x) \psi_{\lambda^{(1)}}(x) \psi_{\lambda^{(2)}}(x) \ldots \psi_{\lambda^{(n)}}(x), \\
J_{\lambda^{(1)} \lambda^{(2)} \ldots \lambda^{(n)}} & =\int d x \psi_{\lambda^{(1)}}(x) \psi_{\lambda^{(2)}}(x) \ldots \psi_{\lambda^{(n)}}(x) .
\end{aligned}
$$

The quadratic coefficients read

$$
A_{\lambda_{R}^{u}, \lambda_{R}^{u \prime} \lambda_{R}^{u \prime \prime}}^{\lambda_{T}^{u}, \lambda^{u \prime} \lambda^{u \prime \prime}}=f_{\lambda_{T}^{u \prime \prime}} f_{\lambda_{R}^{u \prime \prime}} I_{\lambda_{T}^{u \prime} \lambda_{T}^{u \prime \prime}}^{\lambda_{u}^{u}} I_{\lambda_{R}^{u \prime} \lambda_{R}^{u \prime \prime}}^{\lambda_{R}^{u}}
$$

whereas the cubic coefficients are

$$
\begin{aligned}
& B_{\lambda_{R}^{u}, \lambda_{R}^{u} \lambda_{R}^{u \prime \prime} \lambda_{R}^{u \prime \prime \prime}}^{\lambda_{T}^{u}, \lambda^{u \prime} \lambda^{u \prime \prime} \lambda^{u \prime \prime \prime}}=-\frac{1}{2} f_{\lambda_{T}^{u \prime \prime \prime}} f_{\lambda_{R}^{u \prime \prime \prime}}\left(\frac{1}{M_{R}} I_{\lambda_{T}^{u} \lambda_{T}^{u \prime \prime} \lambda_{T}^{u \prime \prime \prime}}^{\lambda_{T}^{u}} \delta_{\lambda_{R}^{u} \lambda_{R}^{u \prime}} J_{\lambda_{R}^{u \prime \prime} \lambda_{R}^{u \prime \prime \prime}}+\frac{1}{M_{T}} I_{\lambda_{R}^{u} \lambda_{R}^{u \prime \prime} \lambda_{R}^{u \prime \prime \prime}}^{\lambda^{u}} \delta_{\lambda_{T}^{u} \lambda_{T}^{u}}{ }^{u}\right. \\
& \left.\times J_{\lambda_{T}^{u \prime \prime} \lambda_{T}^{u \prime \prime \prime}}\right)+\left\{\left[f_{\lambda_{T}} f_{\lambda_{R}}+f_{\lambda_{T}^{u \prime}} f_{\lambda_{R}^{u}{ }^{\prime}}\right] I_{\lambda_{T}^{u \prime} \lambda_{T}}^{\lambda_{T}^{u}} I_{\lambda_{R}^{u} \lambda_{R}}^{\lambda_{u^{u}}^{u}}-\frac{1}{2}\left[\frac{1}{\sqrt{M_{T}}} \delta_{\lambda_{T} 0} \delta_{\lambda_{T}^{u} \lambda_{T}^{u \prime}}\left(1+f_{\lambda_{R}}\right) I_{\lambda_{R}^{u} \lambda_{R}}^{\lambda_{R}^{u}}\right.\right. \\
& \left.\left.+\frac{1}{\sqrt{M_{R}}} \delta_{\lambda_{R} 0} \delta_{\lambda_{R}^{u} \lambda_{R}^{u \prime}}\left(1+f_{\lambda_{T}}\right) I_{\lambda_{T}^{u} \lambda_{T}}^{\lambda_{T}^{u}}\right]\right\} H_{\lambda_{T}^{u \prime \prime} \lambda_{R}^{u \prime \prime}, \lambda_{T}^{u \prime \prime \prime} \lambda_{R}^{u \prime \prime \prime}}^{\lambda_{T} \lambda_{R}} .
\end{aligned}
$$

As is common in synergetics, the cubic coefficients (13) consist in general of two parts, one stemming from the order parameters themselves and the other representing the influence of the center manifold $H$ on the order parameter dynamics according to

$$
S_{\lambda_{T} \lambda_{R}}=H_{\lambda_{T}^{u} \lambda_{R}^{u}, \lambda_{T}^{u \prime} \lambda_{R}^{u}{ }^{\prime \prime}}^{\lambda_{T} \lambda_{\lambda_{T}}^{u} \lambda_{R}^{u}} U_{\lambda_{T}^{u \prime} \lambda_{R}^{u \prime}}
$$

Here the center manifold coefficients $H_{\lambda_{T}^{u} \lambda_{R}^{u}, \lambda_{T}^{u \prime} \lambda_{R}^{u}}^{\lambda_{T} \lambda_{R}}$ are defined by

$$
\begin{aligned}
& H_{\lambda_{T}^{u} \lambda_{R}^{u}, \lambda_{T}^{u} \lambda^{\prime} \lambda_{R}^{\prime \prime}}^{\lambda_{T} \lambda_{R}}=\frac{f_{\lambda_{T}^{u \prime}} f_{\lambda_{R}^{u \prime}}}{\Lambda_{\lambda_{T}^{u} \lambda_{R}^{u}}+\Lambda_{\lambda_{T}^{u} \lambda_{R}^{u}{ }^{\prime}}-\Lambda_{\lambda_{T} \lambda_{R}}}\left[I_{\lambda_{T}^{u} \lambda_{T}^{u}}^{\lambda_{T}} I_{\lambda_{R}^{u} \lambda_{R}^{u \prime}}^{\lambda_{R}}\right. \\
& \left.-\frac{1}{2}\left(\frac{1}{\sqrt{M_{T}}} J_{\lambda_{T}^{u} \lambda_{T}^{u}{ }^{\prime}} I_{\lambda_{R}^{u} \lambda_{R}^{u}}^{\lambda_{R}} \delta_{\lambda_{T} 0}+\frac{1}{\sqrt{M_{R}}} J_{\lambda_{R}^{u} \lambda_{R}^{u}} I_{\lambda_{T}^{u} \lambda_{T}^{u}}^{\lambda_{T}} \delta_{\lambda_{R} 0}\right)\right] .
\end{aligned}
$$

The order parameter equations (9) for the generalized Häussler-von der Malsburg equations (1) can now serve as a starting point for analysing the self-organized formation of retinotopic projections between manifolds of different geometry. 


\section{Strings}

In this section we specialize the generic order parameter equations (9) to one-dimensional Euclidean manifolds of strings with different lengths $L_{T}$ and $L_{R}$. We start with introducing the eigenfunctions in Sect. 3.1. In Sect. 3.2 we observe that the quadratic term vanishes and derive selection rules for the appearance of cubic terms. In this way we essentially simplify the calculation of order parameter equations as compared with [3]. Furthermore, we show that the order parameter equations represent a potential dynamics, and determine the underlying potential in Sect.3.3. A subsequent transformation from complex to real order parameters in Sect. 3.4 leads to constant phase-shift angles. Thus, in Sect. 3.5 we reduce the order parameter dynamics to two variables which correspond to the amplitudes of two diagonal modes. These two modes compete with each other, until one of them vanishes. Within the potential picture this means that the stable uniform state becomes unstable and the system settles in one of the two potential minima, as is discussed in Sect.3.7. After one of the diagonal modes has won, only such modes are excited which contribute to the sharpening of the diagonal. Approximately solving the Häussler-von der Malsburg equations in Sect. 3.8 leads to the following scenario: Above a critical global growth rate $\alpha_{c}$ the uniform state $w_{0}(t, r)=1$ is stable. By decreasing the control parameter $\alpha$, the projection gets sharper and sharper. Finally, if there is no global growth rate of new synapses any more, i.e. $\alpha=0$, the connection weights are given by Dirac's delta function. Thus, a perfect one-to-one retinotopic state is realized. We conclude this discussion of strings with comparing our results with the corresponding analysis of discrete linear chains in Sect. 3.9.

\subsection{Eigenfunctions}

The magnitudes of the manifolds $\mathcal{M}_{T}$ and $\mathcal{M}_{R}$ are given by $M_{T}=L_{T}$ and $M_{R}=L_{R}$, respectively. To avoid problems at the boundaries, we assume periodic boundary conditions, i.e. we consider retina and tectum to be rings with circumferences $L_{T}$ and $L_{R}$, respectively. The eigenvalue problem of the LaplaceBeltrami operator for both manifolds reads

$$
\frac{\partial^{2}}{\partial x^{2}} \psi_{\lambda}(x)=\chi_{\lambda} \psi_{\lambda}(x)
$$

with $x=t, r$, respectively. Using the boundary condition $\psi_{\lambda}(x)=\psi_{\lambda}(x+L)$, this is solved by the eigenfunctions

$$
\psi_{\lambda}(x)=\frac{1}{\sqrt{L}} \exp \left(i \frac{2 \pi}{L} \lambda x\right),
$$

where the eigenvalues are given by $\chi_{\lambda}=-4 \pi^{2} \lambda^{2} / L^{2}$, with $x \in[0, L)$ and $\lambda=0, \pm 1, \pm 2, \ldots$ Every eigenvalue $\chi_{\lambda}$, apart from the special case $\chi_{0}=0$, is two-fold degenerate. The eigenfunctions form a complete orthonormal system:

$$
\int_{0}^{L} d x \psi_{\lambda}(x) \psi_{\lambda^{\prime}}^{*}(x)=\delta_{\lambda \lambda^{\prime}}, \quad \sum_{\lambda=-\infty}^{\infty} \psi_{\lambda}(x) \psi_{\lambda}^{*}\left(x^{\prime}\right)=\delta\left(x-x^{\prime}\right) .
$$

Note that the orthonormality relation in (18) follows directly by inserting (17), whereas the completeness relation is proven by taking into account the Poisson formula [7]. The cooperativity functions only depend on the distance, which is given by the Euclidean distance $\left|x-x^{\prime}\right|$, i.e. $c\left(x, x^{\prime}\right)=c\left(x-x^{\prime}\right)$. Their expansion in terms of the eigenfunctions (17) corresponds to the Fourier series

$$
c\left(x-x^{\prime}\right)=\frac{1}{L} \sum_{\lambda=-\infty}^{\infty} f_{\lambda} \exp \left[i \frac{2 \pi}{L} \lambda\left(x-x^{\prime}\right)\right] .
$$

The expansion coefficients $f_{\lambda}$ are independent of the sign of the parameters $\lambda$, i.e. $f_{\lambda}=f_{-\lambda}$, as the cooperativity functions are symmetric with respect to their arguments: $c\left(x-x^{\prime}\right)=c\left(x^{\prime}-x\right)$. 


\subsection{Synergetic analysis}

To specialize the order parameter equations (9) to the case of strings, we have to determine the integrals (10) and (11) of products of eigenfunctions. With (17) we obtain

$$
\begin{aligned}
& I_{\lambda^{(1)} \lambda^{(2)} \ldots \lambda^{(n)}}^{\lambda}=\left(\frac{1}{\sqrt{L}}\right)^{n-1} \delta_{\lambda^{(1)}+\lambda^{(2)}+\ldots+\lambda^{(n)}, \lambda}, \\
& J_{\lambda^{(1)} \lambda^{(2)} \ldots \lambda^{(n)}}=\left(\frac{1}{\sqrt{L}}\right)^{n-2} \delta_{\lambda^{(1)}+\lambda^{(2)}+\ldots+\lambda^{(n)}, 0} .
\end{aligned}
$$

From these results one can immediately read off the special cases

$$
I_{\lambda^{\prime} \lambda^{\prime \prime}}^{\lambda}=\frac{1}{\sqrt{L}} \delta_{\lambda^{\prime}+\lambda^{\prime \prime}, \lambda}, \quad I_{\lambda^{\prime} \lambda^{\prime \prime} \lambda^{\prime \prime \prime}}^{\lambda}=\frac{1}{L} \delta_{\lambda^{\prime}+\lambda^{\prime \prime}+\lambda^{\prime \prime \prime}, \lambda}, \quad J_{\lambda^{\prime} \lambda^{\prime \prime}}=\delta_{\lambda^{\prime},-\lambda^{\prime \prime}} .
$$

In (12) the first integral of (22) occurs only with unstable values of $\lambda$. As they can only differ by their sign, it follows $\lambda^{\prime}+\lambda^{\prime \prime} \neq \lambda$. Thus, we have $I_{\lambda^{\prime} \lambda^{\prime \prime}}^{\lambda}=0$, i.e. the quadratic term (12) in (9) vanishes: $A_{\lambda_{R}^{u}, \lambda_{R}^{u} \lambda_{R}^{u} \lambda_{R}^{u \prime}}^{\lambda^{\prime \prime} \lambda^{u \prime \prime}}=0$. To arrive at a more concise representation, we split the cubic contribution in (9) in two terms according to

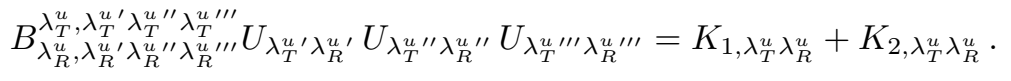

The first term $K_{1}$ takes into account the contribution of the order parameters themselves, while the second term $K_{2}$ represents the influence of the center manifold on the order parameter dynamics. Applying the integrals (22) leads to selection rules for the appearance of cubic terms. It turns out that only those sums lead to non-vanishing contributions where the sum of three unstable modes $\lambda^{u^{\prime}}+\lambda^{u^{\prime \prime}}+\lambda^{u^{\prime \prime \prime}}$ coincides with another unstable mode $\lambda^{u^{\prime}}$. Thus, both for retina and tectum only the following combinations are allowed: $\left(\lambda^{u^{\prime}}, \lambda^{u^{\prime \prime}}, \lambda^{u^{\prime \prime \prime}}\right)=\left(\lambda^{u}, \lambda^{u},-\lambda^{u}\right),\left(\lambda^{u},-\lambda^{u}, \lambda^{u}\right),\left(-\lambda^{u}, \lambda^{u}, \lambda^{u}\right)$. With this selection rule the first cubic term is given by

$$
\begin{aligned}
K_{1, \lambda_{T}^{u} \lambda_{R}^{u}}= & -\frac{f_{\lambda_{T}^{u}}^{T} f_{\lambda_{R}^{u}}^{R}}{2 L_{T} L_{R}}\left[U_{\lambda_{T}^{u} \lambda_{R}^{u^{\prime}}} U_{\lambda_{T}^{u^{\prime \prime}} \lambda_{R}^{u^{\prime \prime}}} U_{-\lambda_{T}^{u^{\prime \prime}} \lambda_{R}^{u^{\prime \prime \prime}}} \delta_{\lambda_{R}^{u^{\prime}}+\lambda_{R}^{u^{\prime \prime}}+\lambda_{R}^{u^{\prime \prime \prime}}, \lambda_{R}^{u}}\right. \\
& \left.+U_{\lambda_{T}^{u^{\prime}} \lambda_{R}^{u}} U_{\lambda_{T}^{u^{\prime \prime}} \lambda_{R}^{u^{\prime \prime}}} U_{\lambda_{T}^{u^{\prime \prime \prime}}-\lambda_{R}^{u^{\prime \prime}}} \delta_{\lambda_{T}^{u^{\prime}}+\lambda_{T}^{u^{\prime \prime}}+\lambda_{T}^{u^{\prime \prime \prime}}, \lambda_{T}^{u}}\right]
\end{aligned}
$$

and the second cubic term reads

$$
\begin{gathered}
K_{2, \lambda_{T}^{u} \lambda_{R}^{u}}=\frac{H_{\lambda_{T}^{\prime} \lambda_{R}^{\prime}, \lambda_{T}^{u^{\prime \prime}} \lambda_{R}^{u^{\prime \prime}} \lambda_{T}^{u^{\prime \prime \prime}} \lambda_{R}^{u^{\prime \prime \prime}}}}{\sqrt{L_{T} L_{R}}} U_{\lambda_{T}^{u^{\prime}} \lambda_{R}^{u^{\prime}}} U_{\lambda_{T}^{u^{\prime \prime}} \lambda_{R}^{u^{\prime \prime}}} U_{\lambda_{T}^{u^{\prime \prime \prime}} \lambda_{R}^{u^{\prime \prime \prime}}}\left\{\left(f_{\lambda_{T}^{\prime}}^{T} f_{\lambda_{R}^{\prime}}^{R}+f_{\lambda_{T}^{u}}^{T} f_{\lambda_{R}^{u}}^{R}\right) \delta_{\lambda_{T}^{u^{\prime}}+\lambda_{T}^{\prime}, \lambda_{T}^{u}}\right. \\
\left.\times \delta_{\lambda_{R}^{u^{\prime}}+\lambda_{R}^{\prime}, \lambda_{R}^{u}}-\frac{1}{2}\left[\left(1+f_{\lambda_{R}^{\prime}}^{R}\right) \delta_{\lambda_{R}^{u^{\prime}}+\lambda_{R}^{\prime}, \lambda_{R}^{u}} \delta_{\lambda_{T}^{\prime} 0} \delta_{\lambda_{T}^{u^{\prime}} \lambda_{T}^{u}}+\left(1+f_{\lambda_{T}^{\prime}}^{T}\right) \delta_{\lambda_{T}^{u^{\prime}}+\lambda_{T}^{\prime}, \lambda_{T}^{u}} \delta_{\lambda_{R}^{\prime} 0} \delta_{\lambda_{R}^{u^{\prime}} \lambda_{R}^{u}}\right]\right\} .
\end{gathered}
$$

The latter depends on the center manifold, which follows from (15), and (22) to be

$$
\begin{array}{r}
H_{\lambda_{T} \lambda_{R}, \lambda_{T}^{u^{\prime \prime}} \lambda_{R}^{u^{\prime \prime}} \lambda_{T}^{u^{\prime \prime \prime}} \lambda_{R}^{u^{\prime \prime \prime}}}=\frac{f_{\lambda_{T}^{u}}^{T} f_{\lambda_{R}^{u}}^{R}}{\sqrt{L_{T} L_{R}}\left(2 \Lambda_{\lambda_{T}^{u} \lambda_{R}^{u}}-\Lambda_{\lambda_{T} \lambda_{R}}\right)}\left[\delta_{\lambda_{T}^{u^{\prime \prime}}+\lambda_{T}^{u^{\prime \prime \prime}}, \lambda_{T}} \delta_{\lambda_{R}^{u^{\prime \prime}}+\lambda_{R}^{u^{\prime \prime \prime}}, \lambda_{R}}\right. \\
\left.-\frac{1}{2}\left(\delta_{\lambda_{T}^{u^{\prime \prime}},-\lambda_{T}^{u^{\prime \prime \prime}}} \delta_{\lambda_{R}^{u^{\prime \prime}}+\lambda_{R}^{u^{\prime \prime \prime}}, \lambda_{R}} \delta_{\lambda_{T} 0}+\delta_{\lambda_{R}^{u^{\prime \prime}},-\lambda_{R}^{u^{\prime \prime \prime}}} \delta_{\lambda_{T}^{u^{\prime \prime}}+\lambda_{T}^{u^{\prime \prime \prime}}, \lambda_{T}} \delta_{\lambda_{R} 0}\right)\right] .
\end{array}
$$




\subsection{Complex order parameters}

We can therefore conclude that the order parameter equations for strings have the form

$$
\dot{U}_{\lambda_{T}^{u} \lambda_{R}^{u}}=h_{\lambda_{T}^{u} \lambda_{R}^{u}}\left(U, U^{*}\right)
$$

with the complex function

$$
h_{\lambda_{T}^{u} \lambda_{R}^{u}}\left(U, U^{*}\right)=\Lambda_{\lambda_{T}^{u} \lambda_{R}^{u}} U_{\lambda_{T}^{u} \lambda_{R}^{u}}+A_{\lambda_{T}^{u} \lambda_{R}^{u}} U_{\lambda_{T}^{u} \lambda_{R}^{u}}^{2} U_{-\lambda_{T}^{u}-\lambda_{R}^{u}}+B_{\lambda_{T}^{u} \lambda_{R}^{u}} U_{\lambda_{T}^{u} \lambda_{R}^{u}} U_{-\lambda_{T}^{u} \lambda_{R}^{u}} U_{\lambda_{T}^{u}-\lambda_{R}^{u}} .
$$

Here we have introduced the coefficients

$$
\begin{aligned}
& A_{\lambda_{T}^{u} \lambda_{R}^{u}}=-\frac{\gamma}{L_{T} L_{R}}\left(2-\frac{\gamma+\gamma^{2 \lambda_{T}^{u}, 2 \lambda_{R}^{u}}}{2 \Lambda_{\lambda_{T}^{u} \lambda_{R}^{u}}-\Lambda_{2 \lambda_{T}^{u}, 2 \lambda_{R}^{u}}}\right), \\
& B_{\lambda_{T}^{u} \lambda_{R}^{u}}=-\frac{\gamma}{L_{T} L_{R}}\left[4-\frac{\gamma+\left(\gamma^{2 \lambda_{T}^{u}, 0}-1\right) / 2}{2 \Lambda_{\lambda_{T}^{u} \lambda_{R}^{u}}-\Lambda_{2 \lambda_{T}^{u}, 0}}-\frac{\gamma+\left(\gamma^{0,2 \lambda_{R}^{u}}-1\right) / 2}{2 \Lambda_{\lambda_{T}^{u} \lambda_{R}^{u}}-\Lambda_{0,2 \lambda_{R}^{u}}}\right]
\end{aligned}
$$

with the abbreviations $\gamma^{\lambda_{T} \lambda_{R}}:=f_{\lambda_{T}}^{T} f_{\lambda_{R}}^{R}$ and $\gamma:=\gamma^{\lambda_{T}^{u}, \lambda_{R}^{u}}=f_{\lambda_{T}^{u}}^{T} f_{\lambda_{R}^{u}}^{R}$. Now we turn to the question whether the order parameter equations (27) represent a potential dynamics. To this end we derived in [8] a condition for the order parameter equations which allows one to conclude whether or not such a potential exists. The potential criterion reads

$$
\frac{\partial h_{\lambda_{T}^{u} \lambda_{R}^{u}}\left(U, U^{*}\right)}{\partial U_{\lambda_{T}^{u^{\prime}} \lambda_{R}^{u^{\prime}}}}=\frac{\partial h_{\lambda_{T}^{u^{\prime}} \lambda_{R}^{u^{\prime}}}^{*}\left(U, U^{*}\right)}{\partial U_{\lambda_{T}^{u} \lambda_{R}^{u}}^{*}},
$$

which is, indeed, fulfilled for (28). Furthermore, we derived in [8] the following conditions for determining the underlying potential:

$$
\dot{U}_{\lambda_{T}^{u} \lambda_{R}^{u}}=-\frac{1}{2} \frac{\partial V\left(U, U^{*}\right)}{\partial U_{\lambda_{T}^{u} \lambda_{R}^{u}}^{*}} .
$$

Integrating (32) yields the potential

$$
\begin{aligned}
V\left(U, U^{*}\right)= & -2 \Lambda_{\lambda_{T}^{u} \lambda_{R}^{u}}\left(U_{\lambda_{T}^{u} \lambda_{R}^{u}} U_{-\lambda_{T}^{u}-\lambda_{R}^{u}}+U_{-\lambda_{T}^{u} \lambda_{R}^{u}} U_{\lambda_{T}^{u}-\lambda_{R}^{u}}\right) \\
& -A_{\lambda_{T}^{u} \lambda_{R}^{u}}\left(U_{\lambda_{T}^{u} \lambda_{R}^{u}}^{2} U_{-\lambda_{T}^{u}-\lambda_{R}^{u}}+U_{-\lambda_{T}^{u} \lambda_{R}^{u}}^{2} U_{\lambda_{T}^{u}-\lambda_{R}^{u}}^{2}\right) \\
& -2 B_{\lambda_{T}^{u} \lambda_{R}^{u}} U_{\lambda_{T}^{u} \lambda_{R}^{u}} U_{-\lambda_{T}^{u}-\lambda_{R}^{u}} U_{-\lambda_{T}^{u} \lambda_{R}^{u}} U_{\lambda_{T}^{u}-\lambda_{R}^{u}} .
\end{aligned}
$$

\subsection{Real order parameters}

For technical purposes it has turned out to be useful to work with complex order parameters so far. However, in order to investigate their contribution to a one-to-one mapping between the strings, we have to transform them to real variables. We construct at first the real modes from the eigenfunctions (5), (17) of the linear operator $\hat{L}$ according to

$$
\begin{aligned}
& c_{\lambda_{T} \lambda_{R}}(t, r)=\frac{1}{2}\left[v_{\lambda_{T} \lambda_{R}}(t, r)+v_{-\lambda_{T}-\lambda_{R}}(t, r)\right]=\frac{1}{\sqrt{L_{T} L_{R}}} \cos \left(\frac{2 \pi}{L_{T}} \lambda_{T} t+\frac{2 \pi}{L_{R}} \lambda_{R} r\right), \\
& s_{\lambda_{T} \lambda_{R}}(t, r)=-\frac{i}{2}\left[v_{\lambda_{T} \lambda_{R}}(t, r)-v_{-\lambda_{T}-\lambda_{R}}(t, r)\right]=\frac{1}{\sqrt{L_{T} L_{R}}} \sin \left(\frac{2 \pi}{L_{T}} \lambda_{T} t+\frac{2 \pi}{L_{R}} \lambda_{R} r\right) .
\end{aligned}
$$


These two modes span a real subspace. If we set

$$
a=\rho \cos \psi, \quad b=\rho \sin \psi ; \quad \rho \geq 0, \quad \psi \in(-\pi, \pi],
$$

the following relation results:

$$
a c_{\lambda_{T} \lambda_{R}}(t, r)+b s_{\lambda_{T} \lambda_{R}}(t, r)=\rho \cos \left(\frac{2 \pi}{L_{T}} \lambda_{T} t+\frac{2 \pi}{L_{R}} \lambda_{R} r-\psi\right) .
$$

Thus, the subspace consists of all phase-shifted functions of $\rho c_{\lambda_{T}} \lambda_{R}(t, r)$. Then the modes belonging to the unstable eigenvalue $\left(\lambda_{T}^{u}, \lambda_{R}^{u}\right)$ are given by the modes $c_{\lambda_{T}^{u} \lambda_{R}^{u}}(t, r)$ and $c_{\lambda_{T}^{u}-\lambda_{R}^{u}}(t, r)$ as well as all phaseshifted functions. Rewriting the unstable part (7)

$$
\begin{aligned}
U(t, r)= & U_{\lambda_{T}^{u} \lambda_{R}^{u}} v_{\lambda_{T}^{u} \lambda_{R}^{u}}(t, r)+U_{-\lambda_{T}^{u}-\lambda_{R}^{u}} v_{-\lambda_{T}^{u}-\lambda_{R}^{u}}(t, r) \\
& +U_{\lambda_{T}^{u}-\lambda_{R}^{u}} v_{\lambda_{T}^{u}-\lambda_{R}^{u}}(t, r)+U_{-\lambda_{T}^{u} \lambda_{R}^{u}} v_{-\lambda_{T}^{u} \lambda_{R}^{u}}(t, r)
\end{aligned}
$$

to real modes (34), (35), leads to

$$
U(t, r)=u_{1} c_{\lambda_{T}^{u} \lambda_{R}^{u}}(t, r)+u_{2} s_{\lambda_{T}^{u} \lambda_{R}^{u}}(t, r)+u_{3} c_{\lambda_{T}^{u}-\lambda_{R}^{u}}(t, r)+u_{4} s_{\lambda_{T}^{u}-\lambda_{R}^{u}}(t, r),
$$

with real variables $u_{j}$ :

$$
\begin{aligned}
U_{\lambda_{T}^{u} \lambda_{R}^{u}} & =\left(u_{1}-i u_{2}\right) / 2, \quad U_{-\lambda_{T}^{u}-\lambda_{R}^{u}}=\left(u_{1}+i u_{2}\right) / 2, \\
U_{\lambda_{T}^{u}-\lambda_{R}^{u}} & =\left(u_{3}-i u_{4}\right) / 2, \quad U_{-\lambda_{T}^{u} \lambda_{R}^{u}}=\left(u_{3}+i u_{4}\right) / 2 .
\end{aligned}
$$

Inserting the transformations (40) into the complex potential (33), we obtain the following real potential

$$
\begin{aligned}
V\left(u_{i}\right)= & -\frac{\Lambda_{\lambda_{T}^{u} \lambda_{R}^{u}}}{2}\left(u_{1}^{2}+u_{2}^{2}+u_{3}^{2}+u_{4}^{2}\right)-\frac{A_{\lambda_{T}^{u} \lambda_{R}^{u}}}{16}\left[\left(u_{1}^{2}+u_{2}^{2}\right)^{2}+\left(u_{3}^{2}+u_{4}^{2}\right)^{2}\right] \\
& -\frac{B_{\lambda_{T}^{u} \lambda_{R}^{u}}}{8}\left(u_{1}^{2}+u_{2}^{2}\right)\left(u_{3}^{2}+u_{4}^{2}\right) .
\end{aligned}
$$

The corresponding equations of evolution for the real order parameters are determined from (41) according to $\dot{u}_{j}=-\partial V\left(u_{i}\right) / \partial u_{j}$. They read explicitly

$$
\begin{aligned}
& \dot{u}_{1}=\left[\Lambda_{\lambda_{T}^{u} \lambda_{R}^{u}}+\frac{A_{\lambda_{T}^{u} \lambda_{R}^{u}}}{4}\left(u_{1}^{2}+u_{2}^{2}\right)+\frac{B_{\lambda_{T}^{u} \lambda_{R}^{u}}}{4}\left(u_{3}^{2}+u_{4}^{2}\right)\right] u_{1}, \\
& \dot{u}_{2}=\left[\Lambda_{\lambda_{T}^{u} \lambda_{R}^{u}}+\frac{A_{\lambda_{T}^{u} \lambda_{R}^{u}}}{4}\left(u_{1}^{2}+u_{2}^{2}\right)+\frac{B_{\lambda_{T}^{u} \lambda_{R}^{u}}}{4}\left(u_{3}^{2}+u_{4}^{2}\right)\right] u_{2}, \\
& \dot{u}_{3}=\left[\Lambda_{\lambda_{T}^{u} \lambda_{R}^{u}}+\frac{A_{\lambda_{T}^{u} \lambda_{R}^{u}}}{4}\left(u_{3}^{2}+u_{4}^{2}\right)+\frac{B_{\lambda_{T}^{u} \lambda_{R}^{u}}}{4}\left(u_{1}^{2}+u_{2}^{2}\right)\right] u_{3}, \\
& \dot{u}_{4}=\left[\Lambda_{\lambda_{T}^{u} \lambda_{R}^{u}}+\frac{A_{\lambda_{T}^{u} \lambda_{R}^{u}}}{4}\left(u_{3}^{2}+u_{4}^{2}\right)+\frac{B_{\lambda_{T}^{u} \lambda_{R}^{u}}}{4}\left(u_{1}^{2}+u_{2}^{2}\right)\right] u_{4} .
\end{aligned}
$$

\subsection{Constant phase shift angles}

According to eq. (37) the unstable part (39) can be written as a superposition of two diagonal modes of different orientation

$$
U(t, r)=\xi \cos \left[\frac{2 \pi}{L_{T}} \lambda_{T}^{u} t+\frac{2 \pi}{L_{R}} \lambda_{R}^{u} r-\psi\right]+\eta \cos \left[\frac{2 \pi}{L_{T}} \lambda_{T}^{u} t-\frac{2 \pi}{L_{R}} \lambda_{R}^{u} r-\varphi\right]
$$



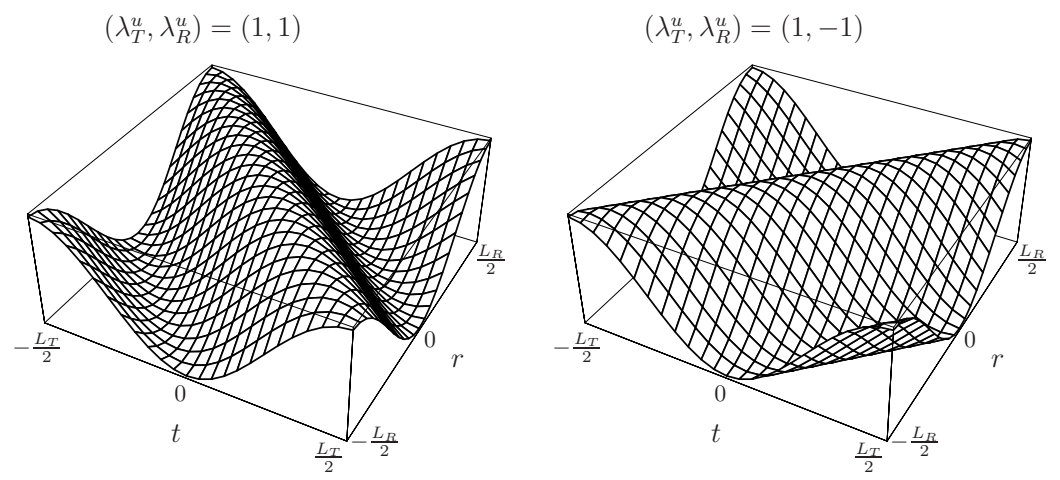

Fig. 2 Diagonal modes of different orientation according to (43) for the case $\left(\lambda_{T}^{u}, \lambda_{R}^{u}\right)=(1,1)$ and $(1,-1)$, respectively. Here the phase shifts are set to $\psi=\varphi=0$.

as is illustrated in Fig. 2. With (36) and (37) we have $u_{1}=\xi \cos \psi, u_{2}=\xi \sin \psi, u_{3}=\eta \cos \varphi, u_{4}=$ $\eta \sin \varphi$. Then the amplitudes of the phase-shift diagonal modes read

$$
\xi=\sqrt{u_{1}^{2}+u_{2}^{2}}, \quad \eta=\sqrt{u_{3}^{2}+u_{4}^{2}}
$$

and the phase angles are given by $\tan \psi=u_{1} / u_{2}, \tan \varphi=u_{3} / u_{4}$. From the order parameter equations (42) it follows $\dot{u}_{1} / \dot{u}_{2}=u_{1} / u_{2}, \dot{u}_{3} / \dot{u}_{4}=u_{3} / u_{4}$. Thus, performing a separation of variables and a subsequent integration leads to the relation $u_{1} / u_{2}=$ const, $u_{3} / u_{4}=$ const. Consequently, the four real equations (42) are reduced to two equations for the mode amplitudes $\xi$ and $\eta$ :

$$
\begin{aligned}
& \dot{\xi}=\left(\Lambda_{\lambda_{T}^{u} \lambda_{R}^{u}}+\frac{A_{\lambda_{T}^{u} \lambda_{R}^{u}}}{4} \xi^{2}+\frac{B_{\lambda_{T}^{u} \lambda_{R}^{u}}}{4} \eta^{2}\right) \xi, \\
& \dot{\eta}=\left(\Lambda_{\lambda_{T}^{u} \lambda_{R}^{u}}+\frac{A_{\lambda_{T}^{u} \lambda_{R}^{u}}}{4} \eta^{2}+\frac{B_{\lambda_{T}^{u} \lambda_{R}^{u}}}{4} \xi^{2}\right) \eta .
\end{aligned}
$$

The corresponding potential is

$$
V(\xi, \eta)=-\frac{\Lambda_{\lambda_{T}^{u} \lambda_{R}^{u}}}{2}\left(\xi^{2}+\eta^{2}\right)-\frac{A_{\lambda_{T}^{u} \lambda_{R}^{u}}}{16}\left(\xi^{4}+\eta^{4}\right)-\frac{B_{\lambda_{T}^{u} \lambda_{R}^{u}}}{8} \xi^{2} \eta^{2} .
$$

Thus, we have reduced the four complex order parameter equations (27), (28) to two real order parameter equations (45) with the potential (46).

\subsection{Monotonous cooperativity functions}

So far our considerations are valid for arbitrary unstable modes $\left(\lambda_{T}^{u}, \lambda_{R}^{u}\right)$. According to the eigenvalue spectrum (6) the unstable modes are determined by the expansion coefficients $f_{\lambda}$ of the cooperativity functions. We therefore derive in this subsection some basic properties of these coefficients. In particular, we investigate the consequences of monotonically decreasing cooperativity functions for their expansion coefficients $f_{\lambda}$. As $c(x)$ is positive and normalized, we conclude $\left|f_{\lambda}\right| \leq 1$. Using the Euler formula, the symmetry $c(x)=c(-x)$, and integrating by parts, the expansion coefficients can be written in the form

$$
f_{\lambda}=-\frac{L}{\pi \lambda} \int_{0}^{L / 2} c^{\prime}(x) \sin \left(\frac{2 \pi}{L} \lambda x\right) d x
$$



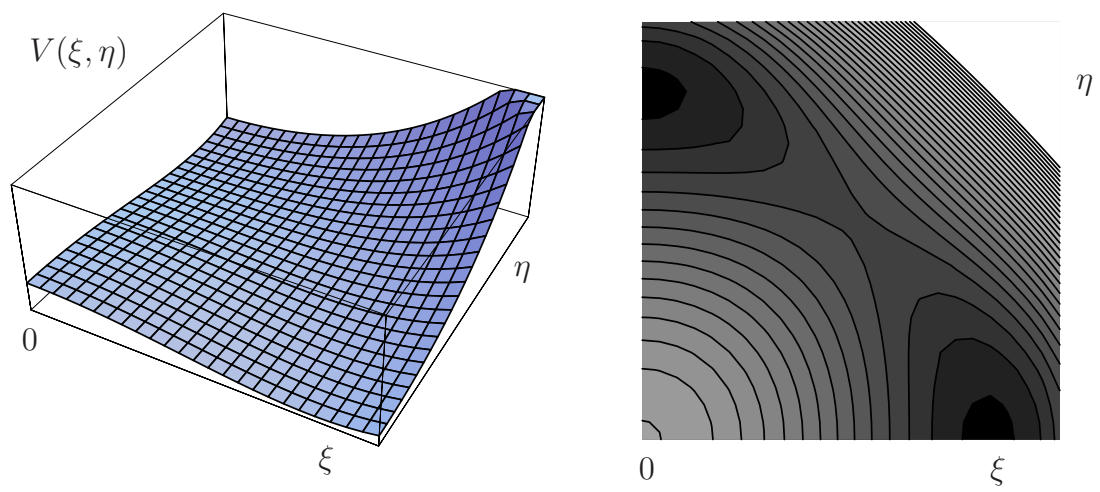

Fig. 3 (online colour at: www.ann-phys.org) The potential $V(\xi, \eta)$ according to eq. (49) with $\Lambda>0$. The originally stable state $\xi=\eta=0$ becomes unstable. The system settles into one of the two minima, i.e. one of the two modes vanishes. The right plot shows the equipotential lines. Dark grey values correspond to small values of the potential $V$.

which makes the symmetry $f_{\lambda}=f_{-\lambda}$ manifest. If we assume monotonically decreasing cooperativity functions, i.e. $d c / d x<0$ for $x \in[0, L / 2]$, we obtain $f_{1}>0$. Furthermore, we can show that $f_{1}$ is the largest expansion coefficient by considering the expression

$$
f_{1}-f_{\lambda}=-\frac{L}{\pi} \int_{0}^{L / 2} c^{\prime}(x)\left[\sin \left(\frac{2 \pi}{L} x\right)-\frac{1}{\lambda} \sin \left(\frac{2 \pi}{L} \lambda x\right)\right] d x .
$$

Because of $c^{\prime}(x)<0$ it follows indeed $f_{1}-f_{\lambda}>0 \quad \forall \lambda \neq 0, \pm 1$. Together with $\left|f_{\lambda}\right|<1$ the maximum eigenvalue of (6) results to be $\Lambda_{\max }=-\alpha+f_{1}^{T} f_{1}^{R}$. Hence in this case there are four unstable modes $\left(\lambda_{T}^{u}, \lambda_{R}^{u}\right)=( \pm 1, \pm 1)$, which corresponds to the result obtained in [3]. However, the most fundamental insight of our more general analysis is that the real order parameter equations (45) are also valid in the case where the cooperativity functions are not monotonic so that any mode $\left(\lambda_{T}^{u}, \lambda_{R}^{u}\right)$ can become unstable. It is plausible that there is a pathological development in animals which corresponds to this case.

\subsection{Potential properties}

We now analyze the properties of the potential (46). For this purpose we restrict ourselves from now on to the unstable modes $\left(\lambda_{T}^{u}, \lambda_{R}^{u}\right)=( \pm 1, \pm 1)$, whose indices will be discarded for the sake of simplicity. Then the potential (46) reads

$$
V(\xi, \eta)=-\frac{\Lambda}{2}\left(\xi^{2}+\eta^{2}\right)-\frac{A}{16}\left(\xi^{4}+\eta^{4}\right)-\frac{B}{8} \xi^{2} \eta^{2},
$$

where the coefficients $A, B$ follow from (29), (30) to be

$$
\begin{aligned}
& A=-\frac{\gamma}{L_{T} L_{R}}\left(2-\frac{\gamma+\gamma^{2,2}}{2 \Lambda-\Lambda_{2,2}}\right), \\
& B=-\frac{\gamma}{L_{T} L_{R}}\left[4-\frac{\gamma+\left(\gamma^{2,0}-1\right) / 2}{2 \Lambda-\Lambda_{2,0}}-\frac{\gamma+\left(\gamma^{0,2}-1\right) / 2}{2 \Lambda-\Lambda_{0,2}}\right] .
\end{aligned}
$$

From the condition $\nabla V=0$ we determine the extrema of $V(\xi, \eta)$ and assign them to a minimum, a maximum, or a saddle point. In the unstable region with $\Lambda>0$ the potential $V(\xi, \eta)$, which is depicted in Fig. 3, has 
- a relative maximum at $P_{1}(0,0)$,

- two relative minima at $P_{2}(0, \sqrt{-4 \Lambda / A})$ and $P_{3}(\sqrt{-4 \Lambda / A}, 0)$,

- a saddle point at $P_{4}(\sqrt{-4 \Lambda /(A+B)}, \sqrt{-4 \Lambda /(A+B)})$.

In the stable region with $\Lambda<0$ only the relative minimum $\xi=\eta=0$ does exist. Initially, the system is in the stable uniform state $w_{0}(t, r)=1$. This state becomes unstable if the control parameter $\alpha$ is decreased to the critical value $\alpha_{c}=f_{1}^{T} f_{1}^{R}$. The eigenvalue $\Lambda_{\max }=-\alpha+f_{1}^{T} f_{1}^{R}$ becomes positive, and the minimum passes into a maximum. The system settles into one of the two equivalent minima, i.e. a symmetry breaking takes place. Thereby the two modes compete with each other and, subsequently, one of the two modes vanishes. Which of them vanishes depends on the initial conditions of $\xi$ and $\eta$. If the condition $\eta(0)>\xi(0)$ is fulfilled, the $\xi$-mode vanishes, and vice versa.

\subsection{One-to-one retinotopy}

In the following we assume that, according to the potential dynamics discussed above, only one of the two modes remains. These two modes show a pronounced maximum for $t=-r$ and $t=r$, respectively, as is shown in Fig. 2. To assess the influence of higher modes, we calculate the center manifold $S(U)$ for the case $\xi=0$ and $\eta \neq 0$ and set $u_{4}=0$ without loss of generality. Then it follows from (44) that $\eta=u_{3}$, and we obtain for the unstable part (39)

$$
U(t, r)=\eta \cos \left(\frac{2 \pi}{L_{T}} t-\frac{2 \pi}{L_{R}} r\right) .
$$

With the center manifold (26) the stable part (8), (14) reads explicitly

$$
S(U)=\frac{2 \gamma}{\sqrt{L_{T} L_{R}}\left(2 \Lambda-\Lambda_{2,2}\right)} \eta^{2} \cos \left(\frac{4 \pi}{L_{T}} t-\frac{4 \pi}{L_{R}} r\right) .
$$

Thus, those modes are excited which strengthen the retinotopic character of the projection. With the help of the complex modes it can be seen that this is also the case for higher modes, i.e. for $\left(\lambda_{T}^{u}, \lambda_{R}^{u}\right)=(1,1)$ exclusively the modes $(2,2),(3,3)$ etc. are excited, which are depicted in Fig. 4. Therefore, we follow [3] and use an ansatz which contains only diagonal modes and insert it into the Häussler-von der Malsburg equations (1). If we restrict ourselves to special cooperativity functions, the resulting recursion relations can be solved analytically by using the method of generating function. Note that our derivation of the solution of the recursion relations corresponds to the gravitating chain in [3].

\subsubsection{Recursion relations}

Motivated by the above remarks we investigate the Häussler-von der Malsburg equations for strings with the ansatz

$$
w(t, r)=\sqrt{L_{T} L_{R}} \sum_{\lambda=-\infty}^{\infty} w_{\lambda} v_{\lambda,-\lambda}(t, r),
$$

where $v_{\lambda,-\lambda}(t, r)$ is defined by (5) and (17). Thus, taking into account the decomposition (19) of the cooperativity functions, the Häussler-von der Malsburg equations (1) can be written as

$$
\dot{w}(t, r)=-\alpha[w(t, r)-1]+w(t, r) \sqrt{L_{T} L_{R}} \sum_{\lambda=-\infty}^{\infty} w_{\lambda} f_{\lambda}^{T} f_{\lambda}^{R} v_{\lambda,-\lambda}(t, r)-p(w) w(t, r),
$$



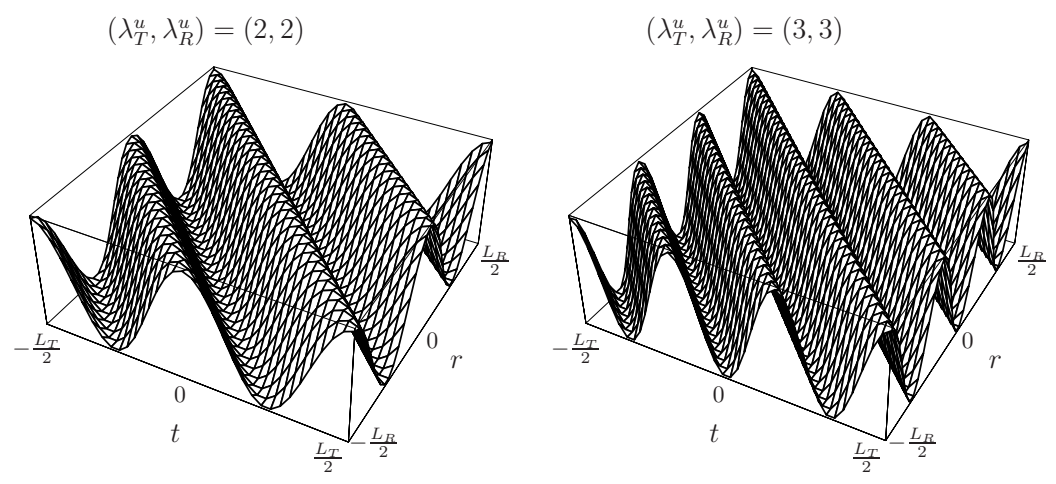

Fig. 4 Higher diagonal modes, which are excited by the unstable mode $\left(\lambda_{T}^{u}, \lambda_{R}^{u}\right)=(1,1)$ of Fig. 2. They amplify the retinotopic character of the $(1,1)$-mode.

where we have introduced the abbreviation

$$
p(w)=\sum_{\lambda=-\infty}^{\infty} w_{-j} w_{j} f_{j}^{T} f_{j}^{R} .
$$

Inserting the ansatz (54) into (55) and comparing the coefficients of the linearely independent functions $v_{\lambda,-\lambda}(t, r)$ yields

$$
\begin{aligned}
& \dot{w}_{0}=-[\alpha+p(w)]\left(w_{0}-1\right), \\
& \dot{w}_{\lambda}=-[\alpha+p(w)] w_{\lambda}+\sum_{j=-\infty}^{\infty} w_{\lambda-j} w_{j} f_{j}^{T} f_{j}^{R}, \quad \lambda \neq 0 .
\end{aligned}
$$

As $w(t, r)$ is positive [1], we obtain that $p(w)>0$ and $\alpha+p(w)>0$. Therefore, the stationary state is determined from (57) to be $w_{0}=1$.

\subsubsection{Special cooperativity functions}

We restrict our further considerations to the following form of the cooperativity functions (19): $f_{0}=$ $1, f_{1} \neq 0, f_{j}=0$ for $j \neq 0, \pm 1$. With the abbreviation $\gamma:=f_{1}^{T} f_{1}^{R}$ the previous result (56) can be written as $p(w)=1+2 \gamma w_{1} w_{-1}$, so that the eqs. (58) for the stationary case reduce to the recursion relation

$$
\left(\alpha+2 \gamma w_{1}^{2}\right) w_{\lambda}=\gamma w_{1}\left(w_{\lambda-1}+w_{\lambda+1}\right), \quad \lambda \neq 0 .
$$

\subsubsection{Generating Function}

To solve the recursion relation (59), we define the generating function

$$
E(z)=\sum_{\lambda=-\infty}^{\infty} w_{\lambda} z^{\lambda}
$$

Multiplying (59) with $z^{\lambda}+z^{-\lambda}$ and performing the sum from $\lambda=1$ up to infinity yields to a linear algebraic equation which is solved by

$$
E(z)=\frac{\alpha}{\alpha+2 \gamma w_{1}^{2}-\gamma w_{1}\left(z+z^{-1}\right)} .
$$




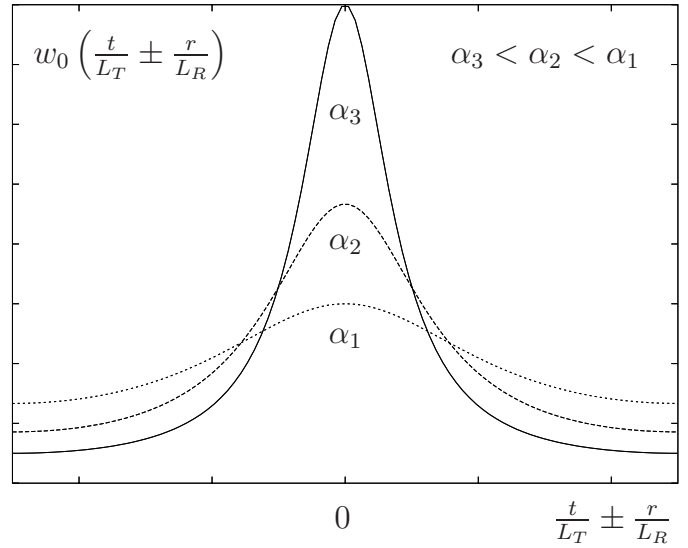

Fig. 5 Decreasing the control parameter $\alpha$ to smaller values, we read off from (64) and (65) that the connection weight converges to Dirac's delta function (66).

To determine the coefficients $w_{\lambda}$ we expand the generating function (61) into a Taylor series:

$$
E(z)=-\frac{\alpha}{\left(\alpha+2 \gamma w_{1}^{2}\right) w\left(z_{1}-z_{1}^{-1}\right)}\left[\sum_{\lambda=1}^{\infty} z_{1}^{\lambda}\left(z^{\lambda}+z^{-\lambda}\right)+1\right], \quad\left|z_{1}\right|<|z|<\left|z_{1}\right|^{-1}
$$

with the abbreviations

$$
w=\frac{\gamma w_{1}}{\alpha+2 \gamma w_{1}^{2}}, \quad z_{1}=\frac{1}{2 w}\left(1+\sqrt{1-4 w^{2}}\right) .
$$

Comparing (62) with (60) by taking into account $w_{0}=1$ determines $w_{1}$ to be

$$
w_{1}=\sqrt{\frac{\gamma-\alpha}{\gamma}}
$$

Thus, together with (63) it follows $z_{1}=w_{1}$, and the remaining coefficients turn out to be $w_{\lambda}=w_{1}^{|\lambda|}$, which is valid not only for $\lambda \neq 0$ but also for $\lambda=0$ due to $w_{0}=1$.

\subsubsection{Limiting cases}

By inserting the latter result into (54) we obtain

$$
w_{0}(t, r)=\frac{1-w_{1}^{2}}{1-2 w_{1} \cos \left(\frac{2 \pi}{L_{T}} t-\frac{2 \pi}{L_{R}} r\right)+w_{1}^{2}} .
$$

For $w_{1}=0$ the stationary uniform state reduces to $w_{0}(t, r)=1 \forall t \in\left[0, L_{T}\right), r \in\left[0, L_{R}\right)$. In the case $w_{1}=1$, i.e. $w_{\lambda}=1 \forall \lambda$, we find with the help of the Poisson formula [7]

$$
w_{0}(t, r)=\delta\left(\frac{t}{L_{T}}-\frac{r}{L_{R}}\right) .
$$

Hence we have a situation which is illustrated in Fig. 5: If the control parameter $\alpha$ is in the neighborhood of $\gamma$, the connection weight is essentially uniform with a small maximum for $t / L_{T}= \pm r / L_{R}$. Further decreasing of $\alpha$ leads to a sharpening of the projection. In the case $\alpha \rightarrow 0$ the projection becomes Dirac's delta function, i.e. a perfect one-to-one retinotopy is achieved. This means that the undifferentiated growth of new synaptic contacts comes to an end when the ordered projection between retina and tectum is fully developed. 


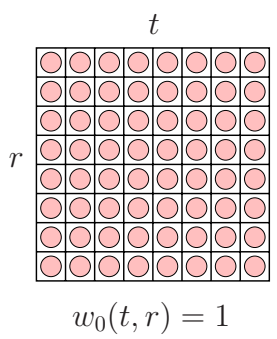

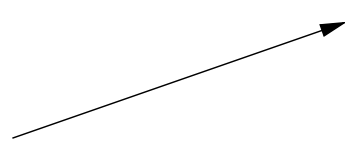

Bifurcation

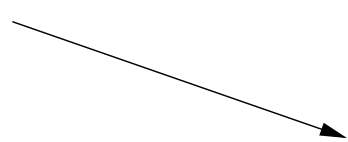

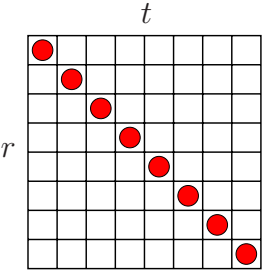

$w_{0}(t, r)=N \delta_{t,-r}$

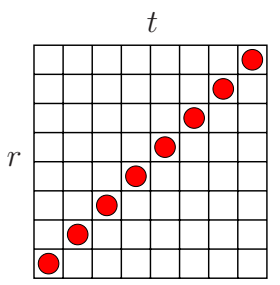

$w_{0}(t, r)=N \delta_{t, r}$

Fig. 6 (online colour at: www.ann-phys.org) Bifurcation in the vicinity of the instability point for the linear chain as analyzed in [3]. The quadratic arrangement of the two linear chains allows a concise representation of the connection weights. Dark gray means high connection weights between the corresponding cells $t$ and $r$. At the uniform initial state all connection weights are equal. The bifurcation drives the system into one of the two possible states, which differ in their orientation. Decreasing the control parameter $\alpha$ to zero leads to a one-to-one retinotopy. Instead of a delta function in the continuous case, here the retinotopic order is described by Kronecker deltas.

\subsection{Comparison with linear chains}

Finally, we compare our results for strings with those of [3] where retina and tectum were treated as linear chains consisting of $N$ cells, respectively. In that reference, the order parameter equations read

$$
\dot{U}_{i j}=\left[\Lambda-\gamma(2-a) U_{i j} U_{-i-j}+\left(4-b^{\prime}-b^{\prime \prime}\right) U_{i-j} U_{-i j}\right] U_{i j}
$$

with the abbreviations

$$
a=-\frac{\gamma+\gamma^{2,2}}{\Lambda_{22}}, \quad b^{\prime}=-\frac{\gamma+\left(\gamma^{2,0}-1\right) / 2}{\Lambda_{20}}, \quad b^{\prime \prime}=-\frac{\gamma+\left(\gamma^{0,2}-1\right) / 2}{\Lambda_{02}} .
$$

The comparison of the coefficients $A, B$ according to (50), (51) with $\gamma(2-a), \gamma\left(4-b^{\prime}-b^{\prime \prime}\right)$ exhibits two differences: the factor $1 / L_{T} L_{R}$ in $A$ and $B$ as well as the term $2 \Lambda$ in the denominator. The absence of the corresponding factor $1 / N^{2}$ in [3] stems from the circumstance that the eigenfunctions were not normalized there. Physically more interesting is the appearance of the term $2 \Lambda$ in the denominator of $A, B$. The reason for this is that we have used the mathematically correct equation for determining the center manifold (15) according to [1,9], whereas in [3] the center manifold is adiabatically approximated by $\dot{S}=0$. However, this ad-hoc method for implementing the adiabatic approximation, which is frequently used in the literature, is only justified for real eigenvalues. As the eigenvalues of the strings are real, we deduce for the vicinity of the instability point the relation $\Lambda=-\alpha+\gamma \approx 0$. Thus, the coefficients (50), (51) turn into those of [3] and the adiabatic approximation $\dot{S}=0$ can be applied here.

Furthermore, our results for the continuous case are analogous to the results for discrete cell arrays. Also the transition to a perfect one-to-one retinotopy takes place in a corresponding way, as is illustrated in 
Fig. 6. Thus, we conclude that our geometry-independent model for the emergence of retinotopic projections developed in [1] contains as a special case the results of [3]. In addition, we have extended the range of validity, i.e. the domain around the instability with $\Lambda=0$, where the order parameter equations represent a quantitatively good approximation, as we have derived a more precise form of the center manifold (15).

\section{Planes}

In this section we extend our discussion to two dimensions where the cell sheets are assumed to be planes of side lengths $L_{1}^{T}, L_{2}^{T}$ and $L_{1}^{R}, L_{2}^{R}$, respectively. To obtain a consistent solution we assume again periodic boundary conditions, i.e. the cell sheets are modelled as surfaces of tori. We start with presenting the linear analysis in Sects. 4.1 and 4.2. Afterwards, in Sects. 4.3 and 4.4 it turns out that we have to calculate in total sixteen order parameter equations where the quadratic term vanishes, as in the case of strings, and where again selection rules reduce the number of cubic terms. This order parameter dynamics turns out to be complicated as the two dimensions do not decouple in a trivial way. Therefore, we have to restrict our analytical discussion of the order parameter dynamics to physiologically interesting special cases. If we set all modes to zero except for one, we find retinotopy only in one dimension, which is shown in Sect. 4.5. In a next step we consider the superposition of two retinotopic modes and investigate the necessary conditions for their coexistence. Such a situation occurs, for instance, when the cooperativity function of the tectum is monotonically decreasing, whereas the cooperativity function of the retina is not monotonic. In Sect. 4.6 we show that taking into account the center manifold contribution or higher modes leads to a sharpening of the retinotopic character of the projection between planar retina and tectum.

\subsection{Eigenfunctions}

In the following we consider both retina and tectum to be planes with side lenghts $L_{1}$ and $L_{2}$. The points on the plane are represented by Cartesian coordinates $x=\left(x_{1}, x_{2}\right), x_{1} \in\left[0, L_{1}\right), x_{2} \in\left[0, L_{2}\right)$. The magnitude of the plane is given by $M=L_{1} L_{2}$. The corresponding eigenvalue equation $\Delta \psi(x)=\chi \psi(x)$ is solved for periodic boundary conditions, i.e. $\psi_{j}\left(x_{j}\right)=\psi_{j}\left(x_{j}+L_{j}\right)$ by the complete orthonormal system of eigenfunctions

$$
\psi_{\lambda}(x)=\frac{1}{\sqrt{L_{1} L_{2}}} \exp \left[2 \pi i\left(\frac{\lambda_{1} x_{1}}{L_{1}}-\frac{\lambda_{2} x_{2}}{L_{2}}\right)\right],
$$

where $\lambda=\left(\lambda_{1}, \lambda_{2}\right)$. The cooperativity function $c\left(x-x^{\prime}\right)$ is expanded according to (3) in this basis:

$$
c\left(x-x^{\prime}\right)=\frac{1}{L_{1} L_{2}} \sum_{\lambda_{1}, \lambda_{2}} f_{\left(\lambda_{1}, \lambda_{2}\right)} \exp \left\{2 \pi i\left[\frac{\lambda_{1}\left(x_{1}-x_{1}^{\prime}\right)}{L_{1}}-\frac{\lambda_{2}\left(x_{2}-x_{2}^{\prime}\right)}{L_{2}}\right]\right\} .
$$

Note that again the expansion coefficients $f_{\left(\lambda_{1}, \lambda_{2}\right)}$ of the cooperativity functions are independent of the signs of the parameters $\lambda_{1}, \lambda_{2}$, as the cooperativity functions should be symmetric with respect to their arguments: $c\left(x-x^{\prime}\right)=c\left(x^{\prime}-x\right)$. This requirement and the linear independence of the exponential functions leads to $f_{\left(\lambda_{1}, \lambda_{2}\right)}=f_{\left( \pm \lambda_{1}, \pm \lambda_{2}\right)}$. From now on we assume that the cooperativity functions decouple with respect to the two dimensions: $c\left(x-x^{\prime}\right)=c_{1}\left(x_{1}-x_{1}^{\prime}\right) c_{2}\left(x_{2}-x_{2}^{\prime}\right)$. As the individual cooperativity functions can be expanded according to

$$
c_{j}\left(x_{j}-x_{j}^{\prime}\right)=\frac{1}{L_{j}} \sum_{\lambda_{j}} f_{\lambda_{j}} \exp \left[i \frac{2 \pi}{L_{j}} \lambda_{j}\left(x_{j}-x_{j}^{\prime}\right)\right], \quad j=1,2,
$$

the decoupling amounts to a factorization of the expansion coefficients: $f_{\left(\lambda_{1}, \lambda_{2}\right)}=f_{\lambda_{1}} f_{\lambda_{2}}$. With this we allow for both isotropic and certain anisotropic cooperativity functions. This is an interesting feature as it is reasonable to assume that real cell sheets have a preferential direction. 


\subsection{Instability point}

We analyze which modes become unstable. According to (6) this depends on the expansion coefficients and the maximum eigenvalues are given by $\Lambda_{\lambda_{T}^{u} \lambda_{R}^{u}}=-\alpha+f_{\lambda_{T}^{u}}^{T} f_{\lambda_{R}^{u}}^{R}$. By doing so we require that all unstable modes become unstable simultaneously. This requirement is due to the fact that the order parameter equations should be approximately valid in the vicinity of the instability point. If the eigenvalues of the corresponding unstable modes would differ significantly, the situation that all modes are in the unstable region would have the consequence that the maximum eigenvalue would be larger than zero, i.e. far away from the instability point. Thus, the order parameter equations would be no adequate approximation. Consequently, we only

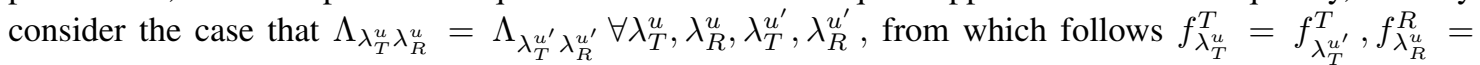
$f_{\lambda_{R}^{u^{\prime}}}^{R} \forall \lambda_{T}^{u}, \lambda_{R}^{u}, \lambda_{T}^{u^{\prime}}, \lambda_{R}^{u^{\prime}}$. However, it is possible that $f_{\lambda_{T}^{u}}^{T} \neq f_{\lambda_{R}^{u}}^{R}$. From now on the unstable modes are assumed to be given by

$$
\lambda^{u}=(1,0),(-1,0),(0,1),(0,-1) .
$$

This occurs, for instance, for monotonically decreasing cooperativity functions where we obtain the relation $f_{1}^{T}>f_{\lambda}^{T}(\lambda \neq 0, \pm 1)$, by analogy with strings (see Sect.3.6). Then the maximum expansion coefficient is given by $f_{\lambda^{u}}=f_{\left(\lambda_{1}^{u}, \lambda_{2}^{u}\right)}=f_{\lambda_{1}^{u}} f_{\lambda_{2}^{u}}$ for $\lambda_{1}^{u}=0, \lambda_{2}^{u}= \pm 1$ and $\lambda_{1}^{u}= \pm 1, \lambda_{2}^{u}=0$, respectively. Note, however, that the unstable modes (72) could also arise for non-monotonic cooperativity functions as we will see below.

\subsection{Order parameter equations}

We specialize the order parameter equations (9) to planes. At first we determine the integrals (10), (11) of products of eigenfunctions (69), which read

$$
\begin{aligned}
& I_{\lambda^{(1)} \lambda^{(2)} \ldots \lambda^{(n)}}^{\lambda}=\left(\frac{1}{L_{1} L_{2}}\right)^{(n-1) / 2} \delta_{\lambda^{(1)}+\lambda^{(2)}+\ldots+\lambda^{(n)}, \lambda}, \\
& J_{\lambda^{(1)} \lambda^{(2)} \ldots \lambda^{(n)}}=\left(\frac{1}{L_{1} L_{2}}\right)^{(n-2) / 2} \delta_{\lambda^{(1)}+\lambda^{(2)}+\ldots+\lambda^{(n)}, 0} .
\end{aligned}
$$

For the order parameter equations (9) we need in (12), (13), (15) the special cases

$$
I_{\lambda^{\prime} \lambda^{\prime \prime}}^{\lambda}=\frac{1}{\sqrt{L_{1} L_{2}}} \delta_{\lambda^{\prime}+\lambda^{\prime \prime}, \lambda}, \quad I_{\lambda^{\prime} \lambda^{\prime \prime} \lambda^{\prime \prime \prime}}^{\lambda}=\frac{1}{L_{1} L_{2}} \delta_{\lambda^{\prime}+\lambda^{\prime \prime}+\lambda^{\prime \prime \prime}, \lambda}, \quad J_{\lambda^{\prime} \lambda^{\prime \prime}}=\delta_{\lambda^{\prime},-\lambda^{\prime \prime}} .
$$

Note that the unstable modes (72) have the property $\lambda^{u^{\prime}}+\lambda^{u^{\prime \prime}} \neq \lambda^{u}$. Thus, the quadratic coefficient (12) vanishes due to the first integral of (75): $A_{\lambda_{R}^{u}, \lambda_{R}^{u} \lambda_{R}^{u \prime \prime}}^{\lambda_{u}^{u}, \lambda^{u \prime} \lambda^{u \prime \prime}}=0$. To yield a concise calculation of the order parameter equations, we introduce modes $\bar{\lambda}^{u}$ which are complementary to the unstable modes $\lambda^{u}$ by permuting the two components:

$$
\bar{\lambda}^{u}= \begin{cases}(0, \pm 1) & \text { if } \lambda^{u}=( \pm 1,0) \\ ( \pm 1,0) & \text { if } \lambda^{u}=(0, \pm 1)\end{cases}
$$

The integrals (75) involve selection rules for the appearance of cubic terms (13) which are analogous to those for strings. This condition turns out to be $\lambda^{u^{\prime}}+\lambda^{u^{\prime \prime}}+\lambda^{u^{\prime \prime \prime}}=\lambda^{u}$ for (72), which leads to the following nine possibilities:

$$
\begin{aligned}
\left(\lambda^{u^{\prime}}, \lambda^{u^{\prime \prime}}, \lambda^{u^{\prime \prime \prime}}\right)= & \left(\lambda^{u}, \lambda^{u},-\lambda^{u}\right),\left(\lambda^{u},-\lambda^{u}, \lambda^{u}\right),\left(-\lambda^{u}, \lambda^{u}, \lambda^{u}\right),\left(\lambda^{u}, \bar{\lambda}^{u},-\bar{\lambda}^{u}\right),\left(\bar{\lambda}^{u}, \lambda^{u},-\bar{\lambda}^{u}\right), \\
& \left(\bar{\lambda}^{u},-\bar{\lambda}^{u}, \lambda^{u}\right),\left(\lambda^{u},-\bar{\lambda}^{u}, \bar{\lambda}^{u}\right),\left(-\bar{\lambda}^{u}, \lambda^{u}, \bar{\lambda}^{u}\right),\left(-\bar{\lambda}^{u}, \bar{\lambda}^{u}, \lambda^{u}\right) .
\end{aligned}
$$


In this way it can be shown that in total 14 possible cubic terms have to be taken into account. The resulting order parameter equations read

$$
\begin{aligned}
& \dot{U}_{\lambda_{T}^{u} \lambda_{R}^{u}}=c_{1}\left(U_{\lambda_{T}^{u} \lambda_{R}^{u}}\right)^{2} U_{-\lambda_{T}^{u}-\lambda_{R}^{u}}+c_{2} U_{\lambda_{T}^{u} \lambda_{R}^{u}} U_{-\lambda_{T}^{u} \lambda_{R}^{u}} U_{\lambda_{T}^{u}-\lambda_{R}^{u}}+c_{3} U_{\lambda_{T}^{u} \lambda_{R}^{u}}\left(U_{\bar{\lambda}_{T}^{u} \lambda_{R}^{u}} U_{-\bar{\lambda}_{T}^{u}-\lambda_{R}^{u}}\right. \\
& \left.+U_{\bar{\lambda}_{T}^{u}-\lambda_{R}^{u}} U_{-\bar{\lambda}_{T}^{u} \lambda_{R}^{u}}\right)+c_{4} U_{\bar{\lambda}_{T}^{u} \lambda_{R}^{u}} U_{\lambda_{T}^{u}-\lambda_{R}^{u}} U_{-\bar{\lambda}_{T}^{u} \lambda_{R}^{u}}+c_{5} U_{\lambda_{T}^{u} \lambda_{R}^{u}}\left(U_{\lambda_{T}^{u} \bar{\lambda}_{R}^{u}} U_{-\lambda_{T}^{u}-\bar{\lambda}_{R}^{u}}+U_{-\lambda_{T}^{u} \bar{\lambda}_{R}^{u}} U_{\lambda_{T}^{u}-\bar{\lambda}_{R}^{u}}\right) \\
& +c_{6} U_{-\lambda_{T}^{u} \lambda_{R}^{u}} U_{\lambda_{T}^{u} \bar{\lambda}_{R}^{u}} U_{\lambda_{T}^{u}-\bar{\lambda}_{R}^{u}}+c_{7} U_{\lambda_{T}^{u} \lambda_{R}^{u}}\left(U_{\bar{\lambda}_{T}^{u} \bar{\lambda}_{R}^{u}} U_{-\bar{\lambda}_{T}^{u}-\bar{\lambda}_{R}^{u}}+U_{\bar{\lambda}_{T}^{u}-\bar{\lambda}_{R}^{u}} U_{-\bar{\lambda}_{T}^{u} \bar{\lambda}_{R}^{u}}\right) \\
& +c_{8}\left(U_{\bar{\lambda}_{T}^{u} \lambda_{R}^{u}} U_{\lambda_{T}^{u} \bar{\lambda}_{R}^{u}} U_{-\bar{\lambda}_{T}^{u}-\bar{\lambda}_{R}^{u}}+U_{-\bar{\lambda}_{T}^{u} \lambda_{R}^{u}} U_{\lambda_{T}^{u} \bar{\lambda}_{R}^{u}} U_{\bar{\lambda}_{T}^{u}-\bar{\lambda}_{R}^{u}}\right. \\
& \left.+U_{\bar{\lambda}_{T}^{u} \lambda_{R}^{u}} U_{\lambda_{T}^{u}-\bar{\lambda}_{R}^{u}} U_{-\bar{\lambda}_{T}^{u} \bar{\lambda}_{R}^{u}}+U_{-\bar{\lambda}_{T}^{u} \lambda_{R}^{u}} U_{\lambda_{T}^{u}-\bar{\lambda}_{R}^{u}} U_{\bar{\lambda}_{T}^{u} \bar{\lambda}_{R}^{u}}\right) .
\end{aligned}
$$

The coefficients $c_{1}-c_{8}$ are fully determined by the expansion coefficients $f_{\lambda}$ of the cooperativity functions and the control parameter $\alpha$, as is documented in [8].

\subsection{Real variables}

To investigate how the complex order parameters contribute to the one-to-one-retinotopy between the planes, we have to transform them to real variables. To this end we introduce the transformation

$$
u_{\lambda_{T}^{u} \lambda_{R}^{u}}=U_{\lambda_{T}^{u} \lambda_{R}^{u}}+U_{-\lambda_{T}^{u}-\lambda_{R}^{u}}, \quad v_{\lambda_{T}^{u} \lambda_{R}^{u}}=i\left(U_{\lambda_{T}^{u} \lambda_{R}^{u}}-U_{-\lambda_{T}^{u}-\lambda_{R}^{u}}\right) .
$$

Thus, with the help of the function

$$
\begin{aligned}
h_{\lambda_{T}^{u} \lambda_{R}^{u}, \lambda_{T}^{u^{\prime}} \lambda_{R}^{u^{\prime}}, \lambda_{T}^{u^{\prime \prime}} \lambda_{R}^{u^{\prime \prime}}}(u, v):= & u_{\lambda_{T}^{u} \lambda_{R}^{u}} u_{\lambda_{T}^{u^{\prime}} \lambda_{R}^{u^{\prime}}} u_{\lambda_{T}^{u^{\prime \prime}} \lambda_{R}^{u^{\prime \prime}}}-u_{\lambda_{T}^{u} \lambda_{R}^{u}} v_{\lambda_{T}^{u^{\prime}} \lambda_{R}^{u^{\prime}}} v_{\lambda_{T}^{u^{\prime \prime}} \lambda_{R}^{u^{\prime \prime}}} \\
& +v_{\lambda_{T}^{u} \lambda_{R}^{u}} u_{\lambda_{T}^{u^{\prime}} \lambda_{R}^{u^{\prime}}} v_{\lambda_{T}^{u^{\prime \prime}} \lambda_{R}^{u^{\prime \prime}}}+v_{\lambda_{T}^{u} \lambda_{R}^{u}} v_{\lambda_{T}^{u^{\prime}} \lambda_{R}^{u^{\prime}}} u_{\lambda_{T}^{u^{\prime \prime}} \lambda_{R}^{u^{\prime \prime}}}
\end{aligned}
$$

the complex order parameter equations (78) are transformed to real ones as follows:

$$
\begin{aligned}
& \dot{u}_{\lambda_{T}^{u} \lambda_{R}^{u}}=u_{\lambda_{T}^{u} \lambda_{R}^{u}}\left[c_{1}\left(u_{\lambda_{T}^{u} \lambda_{R}^{u}}^{2}+v_{\lambda_{T}^{u} \lambda_{R}^{u}}^{2}\right)+c_{2}\left(u_{\lambda_{T}^{u}-\lambda_{R}^{u}}^{2}+v_{\lambda_{T}^{u}-\lambda_{R}^{u}}^{2}\right)\right. \\
& \quad+c_{3}\left(u_{\bar{\lambda}_{T}^{u} \lambda_{R}^{u}}^{2}+v_{\bar{\lambda}_{T}^{u} \lambda_{R}^{u}}^{2}+u_{\bar{\lambda}_{T}^{u}-\lambda_{R}^{u}}^{2}+v_{\bar{\lambda}_{T}^{u}-\lambda_{R}^{u}}^{2}\right)+c_{5}\left(u_{\lambda_{T}^{u} \bar{\lambda}_{R}^{u}}^{2}+v_{\lambda_{T}^{u} \bar{\lambda}_{R}^{u}}^{2}+u_{\lambda_{T}^{u}-\bar{\lambda}_{R}^{u}}^{2}+v_{\lambda_{T}^{u}-\bar{\lambda}_{R}^{u}}^{2}\right) \\
& \left.\quad+c_{7}\left(u_{\bar{\lambda}_{T}^{u} \bar{\lambda}_{R}^{u}}^{2}+v_{\bar{\lambda}_{T}^{u} \bar{\lambda}_{R}^{u}}^{2}+u_{\bar{\lambda}_{T}^{u}-\bar{\lambda}_{R}^{u}}^{2}+v_{\bar{\lambda}_{T}^{u}-\bar{\lambda}_{R}^{u}}^{2}\right)\right]+c_{6} h_{\lambda_{T}^{u}-\lambda_{R}^{u}, \lambda_{T}^{u} \bar{\lambda}_{R}^{u}, \lambda_{T}^{u}-\bar{\lambda}_{R}^{u}}(u, v) \\
& \quad+c_{8}\left[h_{\bar{\lambda}_{T}^{u} \bar{\lambda}_{R}^{u}, \bar{\lambda}_{T}^{u} \lambda_{R}^{u}, \lambda_{T}^{u} \bar{\lambda}_{R}^{u}}(u, v)+h_{\bar{\lambda}_{T}^{u}-\lambda_{R}^{u}, \lambda_{T}^{u} \bar{\lambda}_{R}^{u}, \bar{\lambda}_{T}^{u}-\bar{\lambda}_{R}^{u}}(u, v)\right. \\
& \left.\quad+h_{\bar{\lambda}_{T}^{u}-\bar{\lambda}_{R}^{u}, \lambda_{T}^{u}-\bar{\lambda}_{R}^{u}, \bar{\lambda}_{T}^{u} \lambda_{R}^{u}}(u, v)+h_{\bar{\lambda}_{T}^{u}-\lambda_{R}^{u}, \lambda_{T}^{u}-\bar{\lambda}_{R}^{u}, \bar{\lambda}_{T}^{u} \bar{\lambda}_{R}^{u}}(u, v)\right],
\end{aligned}
$$

The equations for the amplitudes $v_{\lambda_{T}^{u} \lambda_{R}^{u}}$ have an identical structure as they are obtained from (81) by exchanging the variables $u$ and $v$. It can be shown that the order parameter dynamics (81) is governed by a potential [8]. However, as the corresponding expression for the potential is lengthy, we will not discuss it here explicitly. Instead, we investigate different analytical cases which depend on the number of nonvanishing modes. In particular, we are interested in the emergence of retinotopical ordered projections between the planes.

\subsection{Retinotopic projections: non-vanishing modes}

We start with the assumption that only the amplitudes $u_{j}, v_{j}$ of one mode are different from zero. We consider the case $j=2$, but the other cases yield analogous results. The unstable part (7) reads

$$
U(t, r)=U_{10,-10} \exp \left[i 2 \pi\left(\frac{t_{1}}{L_{1}^{T}}-\frac{r_{1}}{L_{1}^{R}}\right)\right]+U_{-10,10} \exp \left[-i 2 \pi\left(\frac{t_{1}}{L_{1}^{T}}-\frac{r_{1}}{L_{1}^{R}}\right)\right],
$$


which is equivalent to

$$
U(t, r)=u_{2} \cos \left[2 \pi\left(\frac{t_{1}}{L_{1}^{T}}-\frac{r_{1}}{L_{1}^{R}}\right)\right]+v_{2} \sin \left[2 \pi\left(\frac{t_{1}}{L_{1}^{T}}-\frac{r_{1}}{L_{1}^{R}}\right)\right] .
$$

The real order parameter equations (81) reduce to

$$
\dot{u}_{2}=\Lambda u_{2}+\frac{c_{1}}{4} u_{2}\left(u_{2}^{2}+v_{2}^{2}\right), \quad \dot{v}_{2}=\Lambda v_{2}+\frac{c_{1}}{4} v_{2}\left(u_{2}^{2}+v_{2}^{2}\right) .
$$

We obtain constant phase-shift angles, which was already discussed in the case of strings (see Sect. 3.5). With $\xi=\sqrt{u_{2}^{2}+v_{2}^{2}}$ it follows $\dot{\xi}=\Lambda \xi+\left(c_{1} / 4\right) \xi^{3}$. For the stationary case this leads to $\xi=0$ or $\xi=\sqrt{-4 \Lambda / c_{1}}$. We are only interested in the case $\xi \neq 0$. This case corresponds to a retinotopy between $r_{1}$ and $t_{1}$, respectively. Thus, we have a retinotopic order only in one dimension and not in the whole plane.

Now we examine the question, if two modes are able to generate a retinotopic state in the plane. As a typical example we consider the case that $u_{2}, v_{2}$ as well as $u_{8}, v_{8}$ remain. Thus, the unstable part (7) has the complex decomposition

$$
\begin{aligned}
U(t, r)= & U_{10,-10} \exp \left[i 2 \pi\left(\frac{t_{1}}{L_{1}^{T}}-\frac{r_{1}}{L_{1}^{R}}\right)\right]+U_{-10,10} \exp \left[-i 2 \pi\left(\frac{t_{1}}{L_{1}^{T}}-\frac{r_{1}}{L_{1}^{R}}\right)\right] \\
& +U_{01,0-1} \exp \left[i 2 \pi\left(\frac{t_{2}}{L_{2}^{T}}-\frac{r_{2}}{L_{2}^{R}}\right)\right]+U_{0-1,01} \exp \left[-i 2 \pi\left(\frac{t_{2}}{L_{2}^{T}}-\frac{r_{2}}{L_{2}^{R}}\right)\right],
\end{aligned}
$$

which due to (79) corresponds to the real decomposition

$$
\begin{aligned}
U(t, r)= & u_{2} \cos \left[2 \pi\left(\frac{t_{1}}{L_{1}^{T}}-\frac{r_{1}}{L_{1}^{R}}\right)\right]+v_{2} \sin \left[2 \pi\left(\frac{t_{1}}{L_{1}^{T}}-\frac{r_{1}}{L_{1}^{R}}\right)\right] \\
& +u_{8} \cos \left[2 \pi\left(\frac{t_{2}}{L_{2}^{T}}-\frac{r_{2}}{L_{2}^{R}}\right)\right]+v_{8} \sin \left[2 \pi\left(\frac{t_{2}}{L_{2}^{T}}-\frac{r_{2}}{L_{2}^{R}}\right)\right] .
\end{aligned}
$$

The real order parameter equations (81) read

$$
\begin{aligned}
& \dot{u}_{2}=\left[\Lambda+\frac{c_{1}}{4}\left(u_{2}^{2}+v_{2}^{2}\right)+\frac{c_{7}}{4}\left(u_{8}^{2}+v_{8}^{2}\right)\right] u_{2}, \\
& \dot{v}_{2}=\left[\Lambda+\frac{c_{1}}{4}\left(u_{2}^{2}+v_{2}^{2}\right)+\frac{c_{7}}{4}\left(u_{8}^{2}+v_{8}^{2}\right)\right] v_{2}, \\
& \dot{u}_{8}=\left[\Lambda+\frac{c_{1}}{4}\left(u_{8}^{2}+v_{8}^{2}\right)+\frac{c_{7}}{4}\left(u_{2}^{2}+v_{2}^{2}\right)\right] u_{8}, \\
& \dot{v}_{8}=\left[\Lambda+\frac{c_{1}}{4}\left(u_{8}^{2}+v_{8}^{2}\right)+\frac{c_{7}}{4}\left(u_{2}^{2}+v_{2}^{2}\right)\right] v_{8} .
\end{aligned}
$$

Again we obtain constant phase-shift angles. With the amplitudes $\xi=\sqrt{u_{2}^{2}+v_{2}^{2}}, \eta=\sqrt{u_{8}^{2}+v_{8}^{2}}$ the following coupled equations result

$$
\dot{\xi}=\left(\Lambda+\frac{c_{1}}{4} \xi^{2}+\frac{c_{7}}{4} \eta^{2}\right) \xi, \quad \dot{\eta}=\left(\Lambda+\frac{c_{1}}{4} \eta^{2}+\frac{c_{7}}{4} \xi^{2}\right) \eta .
$$

We investigate under which conditions the two retinotopic modes coexist. If $\xi, \eta \neq 0$, we obtain from $\dot{\xi}=\dot{\eta}=0$ the relation $\left(\xi^{2}-\eta^{2}\right)\left(c_{1}-c_{7}\right)=0$. As we should minimize the restrictions for the coefficients $c_{1}, c_{7}$, we have in general $c_{1} \neq c_{7}$, so we conclude $\xi=\eta$. Inserting this result in (88) for the stationary case leads to

$$
\xi=\eta=\sqrt{-\frac{4 \Lambda}{c_{1}+c_{7}}} .
$$



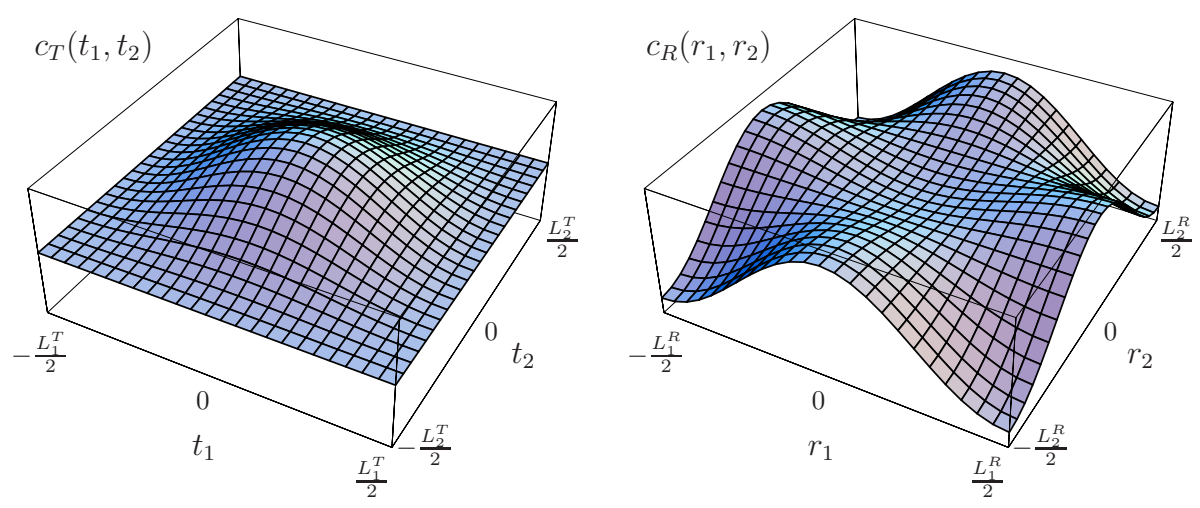

Fig. 7 (online colour at: www.ann-phys.org) Cooperativity functions for retina and tectum according to the special cases (92). The restriction to monotonically decreasing cooperativity functions has to be abandoned.
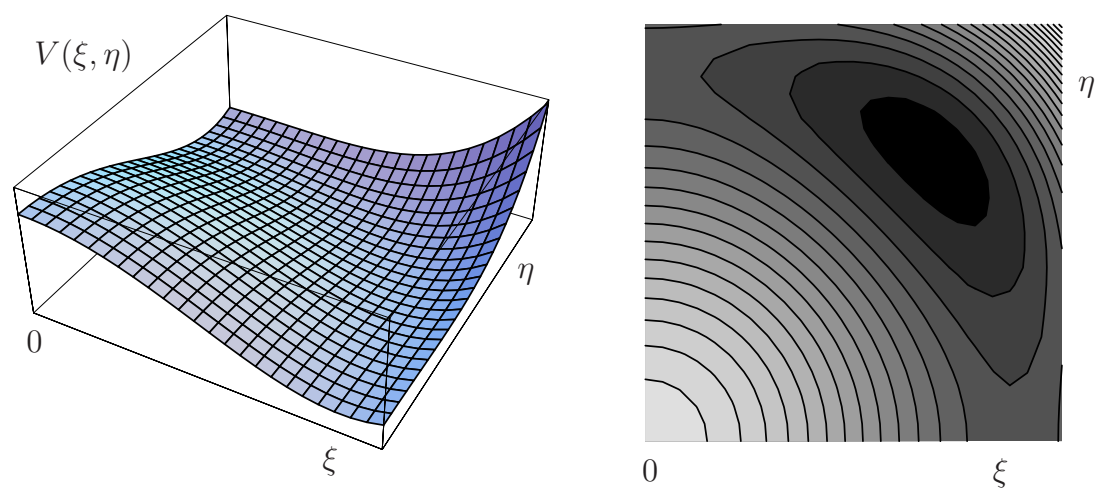

Fig. 8 (online colour at: www.ann-phys.org) The potential (90) for two coexistent retinotopic modes. According to (89) there is a maximum at $\xi=\eta=0$ and a minimum at $\xi=\eta$. The right plot shows the equipotential lines, which pronounces the extrema of $V$.

As the amplitudes $\xi, \eta$ have to be real and $\Lambda>0$, the coefficients $c_{1}, c_{7}$ have to fulfill the condition $c_{1}+c_{7}<0$. Furthermore, we require that the coexistence of both modes is stable. To this end we consider the potential

$$
V(\xi, \eta)=-\frac{\Lambda}{2}\left(\xi^{2}+\eta^{2}\right)-\frac{c_{1}}{16}\left(\xi^{4}+\eta^{4}\right)-\frac{c_{7}}{8} \xi^{2} \eta^{2},
$$

which reproduces according to

$$
\dot{\xi}=-\frac{\partial V(\xi, \eta)}{\partial \xi}, \quad \dot{\eta}=-\frac{\partial V(\xi, \eta)}{\partial \eta}
$$

the amplitude equations (88). A stable state corresponds to a minimum of the potential (90), which leads to the condition $c_{1}<c_{7}$. With the explicit form of the coefficients $c_{1}$ and $c_{7}$ derived in [8] these considerations lead to the result, that either $\gamma^{20,20}$ or $\gamma^{11,11}$ could vanish. If $\gamma^{20,20}=0$, the stability is guaranteed by $-5 \gamma^{11,11} / 3<\gamma<-7 \gamma^{11,11}$, whereas $\gamma^{11,11}=0$ demands $5 \gamma^{20,20} / 3<\gamma<-\gamma^{20,20}$. As an example we consider the first case and assume a special form of the cooperativity functions (70) with the expansion coefficients

$$
f_{ \pm 10}^{T}=f_{0 \pm 1}^{T}=f_{ \pm 10}^{R}=f_{0 \pm 1}^{R}=0.1, \quad f_{ \pm 1, \pm 1}^{T}=0.05, \quad f_{ \pm 1, \pm 1}^{R}=-0.1,
$$



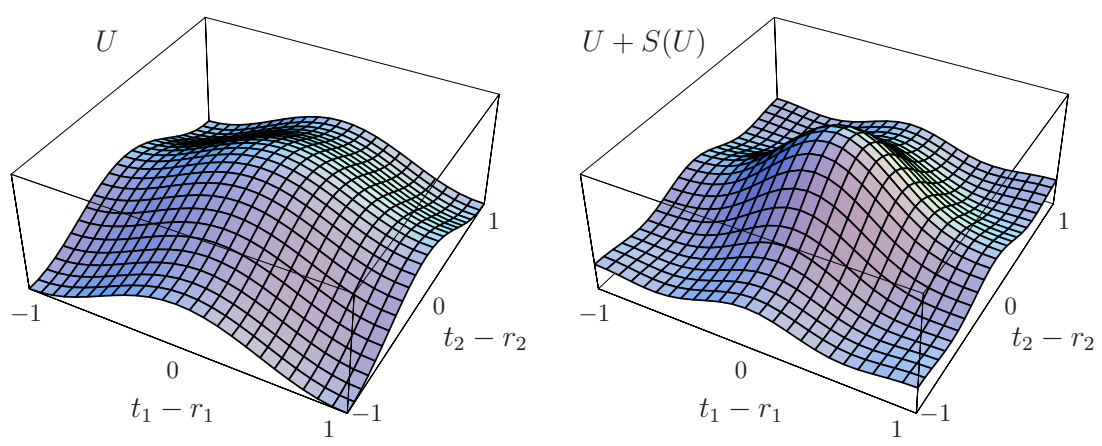

Fig. 9 (online colour at: www.ann-phys.org) The contribution of the center manifold $S(U)$ leads to a more distinct concentration of the connection weight $w(t, r) \approx 1+U(t, r)+S(U(t, r))$ around $t=r$, as compared with the approximation $w(t, r) \approx 1+U(t, r)$.

thus it follows $\gamma=0.01$ and $\gamma^{11,11}=-0.005$. However, although the cooperativity function of the tectum is monotonically decreasing, the cooperativity function of the retina is not monotonic in this case, as is illustrated in Fig. 7. The corresponding potential (90) is shown in Fig. 8.

\subsection{Center manifold}

In the next step we analyze the influence of the center manifold $S(U)$ for the special case (92). The connection weight can be represented as $w(t, r)=1+U(t, r)+S(U(t, r))$ (see Sect.2). Using the relations (14) and (15) the stable part is approximated in the second order. We rewrite the result to real variables and eigenfunctions, where we set $L_{1}^{T, R}=L_{2}^{T, R}=2$. In this way we compare $U(t, r)$ with $U(t, r)+S(U(t, r))$ in Fig.9. It is evident that the retinotopic projection gets sharper due to the contribution of the center manifold, i.e. the projection is maximal around the point $t=r$. This corresponds to the situation found in the case of linear strings; the contribution of the higher modes have the tendency to support the emergence of retinotopic order.

\section{Spheres}

In this Section we apply our general model [1] again to projections between two-dimensional manifolds. Now, however, we consider manifolds with constant positive curvature. Typically, the retina represents approximately a hemisphere, whereas the tectum has an oval form $[2,8]$. Thus, it is biologically reasonable to model both cell sheets by spherical manifolds. Without loss of generality we assume that the two cell sheets for retina and tectum are represented by the surfaces of two unit spheres, respectively. Thus, in our model, the corresponding continuously distributed cells are represented by unit vectors $\hat{r}$ and $\hat{t}$. Every ordered pair $(\hat{t}, \hat{r})$ is connected by a positive connection weight $w(\hat{t}, \hat{r})$ as is illustrated in Fig. 10. The generalized Häussler-von der Malsburg equations (1) for these connection weights are specified as follows

$$
\dot{w}(\hat{t}, \hat{r})=f(\hat{t}, \hat{r}, w)-\frac{w(\hat{t}, \hat{r})}{8 \pi} \quad \int d \Omega_{t^{\prime}} f\left(\hat{t}^{\prime}, \hat{r}, w\right)-\frac{w(\hat{t}, \hat{r})}{8 \pi} \quad \int d \Omega_{r^{\prime}} f\left(\hat{t}, \hat{r}^{\prime}, w\right),
$$

where the total growth rate is defined by

$$
f(\hat{t}, \hat{r}, w)=\alpha+w(\hat{t}, \hat{r}) \int d \Omega_{t^{\prime}} \int d \Omega_{r^{\prime}} c_{T}\left(\hat{t} \cdot \hat{t}^{\prime}\right) c_{R}\left(\hat{r} \cdot \hat{r}^{\prime}\right) w\left(\hat{t}^{\prime}, \hat{r}^{\prime}\right) .
$$

The integrations in (93) and (94) are performed over all points $\hat{t}, \hat{r}$ on the spheres, where $d \Omega_{t}, d \Omega_{r}$ represent the differential solid angles of the corresponding unit spheres. Note that the factors $8 \pi$ in eq. (93) are twice the 


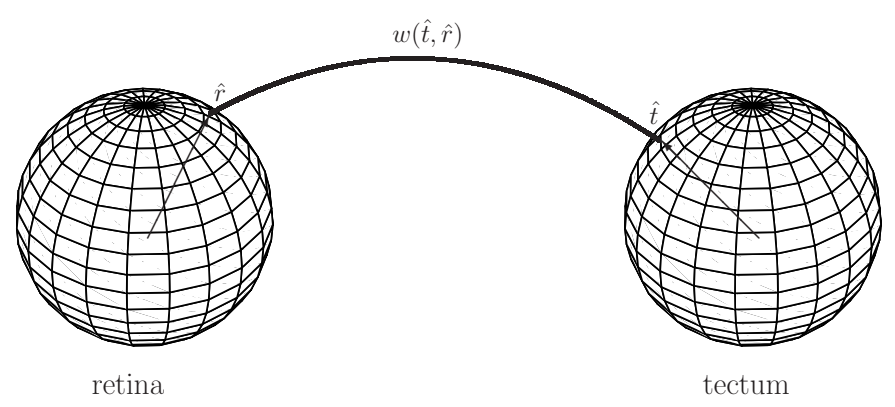

Fig. 10 The cells of retina and tectum, which are assumed to be continuously distributed on unit spheres, are represented by their unit vectors $\hat{r}$ and $\hat{t}$, respectively. The two cell sheets are connected by positive connection weights $w(\hat{t}, \hat{r})$.

measure of the unit sphere. After discussing the linear stability analysis around the homogeneous solution of the generalized Häussler-von der Malsburg equations (93) in Sect. 5.1, we perform the nonlinear synergetic analysis in Sect. 5.2, which yields the underlying order parameter equations in the vicinity of the bifurcation. As in the case of Euclidean manifolds, we show that they have no quadratic terms, represent a potential dynamics, and allow for retinotopic modes. In Sect.5.3 we include the influence of higher modes upon the connection weights, which leads to recursion relations for the corresponding amplitudes. If we restrict ourselves to special cooperativity functions, the resulting recursion relations can be solved analytically by using again the method of generating functions, which was already applied in the case of strings. As a result of our analysis we obtain a perfect one-to-one retinotopy if the global growth rate $\alpha$ is decreased to zero.

\subsection{Linear analysis}

According to the general reasoning in [1] we start with fixing the metric on the manifolds and determine the eigenfunctions of the corresponding Laplace-Beltrami operator. Afterwards, we expand the cooperativity functions with respect to these eigenfunctions and perform a linear analysis of the stationary uniform state.

\subsubsection{Laplace-Beltrami operator}

For the time being we neglect the distinction between retina and tectum, because the following considerations are valid for both manifolds. Using spherical coordinates, we write the unit vector on the sphere as $\hat{x}=$ $(\sin \vartheta \cos \varphi, \sin \vartheta \sin \varphi, \cos \vartheta)$. The Laplace-Beltrami operator for the sphere has the well-known form

$$
\Delta_{\vartheta, \varphi}=\frac{1}{\sin \vartheta} \frac{\partial}{\partial \vartheta}\left(\sin \vartheta \frac{\partial}{\partial \vartheta}\right)+\frac{1}{\sin ^{2} \vartheta} \frac{\partial^{2}}{\partial \varphi^{2}},
$$

whose eigenfunctions are known to be given by spherical harmonics $Y_{l m}(\hat{x})$, i.e.

$$
\Delta_{\vartheta, \varphi} Y_{l m}(\hat{x})=-l(l+1) Y_{l m}(\hat{x}),
$$

which form a complete orthonormal system on the unit sphere.

\subsubsection{Cooperativity functions}

The argument of the cooperativity functions $c\left(\hat{x} \cdot \hat{x}^{\prime}\right)$ is the scalar product $\hat{x} \cdot \hat{x}^{\prime}$ which takes values between -1 and +1 . Therefore the cooperativity functions can be expanded in terms of Legendre functions $P_{l}\left(\hat{x} \cdot \hat{x}^{\prime}\right)$, which form a complete orthogonal system on this interval [10, 7.221.1]:

$$
c\left(\hat{x} \cdot \hat{x}^{\prime}\right)=\sum_{l=0}^{\infty} \frac{2 l+1}{4 \pi} f_{l} P_{l}\left(\hat{x} \cdot \hat{x}^{\prime}\right) .
$$


Using the Legendre addition theorem [13] we arrive, for each manifold, at the expansion (3)

$$
c_{T}\left(\hat{t} \cdot \hat{t}^{\prime}\right)=\sum_{L=0}^{\infty} \sum_{M=-L}^{L} f_{L}^{T} Y_{L M}^{T}(\hat{t}) Y_{L M}^{T *}\left(\hat{t}^{\prime}\right), \quad c_{R}\left(\hat{r} \cdot \hat{r}^{\prime}\right)=\sum_{l=0}^{\infty} \sum_{m=-l}^{l} f_{l}^{R} Y_{l m}^{R}(\hat{r}) Y_{l m}^{R *}\left(\hat{r}^{\prime}\right) .
$$

Note that the normalization of the cooperativity functions and the orthonormality relations lead to the constraints $f_{0}^{T}=f_{0}^{R}=1$.

\subsubsection{Eigenvalues}

According to Sect. 2, a linear stability analysis around the stationary uniform state leads to the eigenvalue problem of the linear operator (4). It has the eigenfunctions $v_{L l}^{M m}(\hat{t}, \hat{r})=Y_{L M}^{T}(\hat{t}) Y_{l m}^{R}(\hat{r})$ and the spectrum of eigenvalues (6). By changing the uniform growth rate $\alpha$ in a suitable way, the real parts of some eigenvalues (6) become positive and the system can be driven to the neighborhood of an instability. Which eigenvalues (6) become unstable in general depends on the respective values of the given expansion coefficients $f_{L}^{T}$, $f_{l}^{R}$. If we assume monotonically decreasing expansion coefficients $f_{L}^{T}, f_{l}^{R}$, i.e.

$$
1=f_{0}^{T} \geq f_{1}^{T} \geq f_{2}^{T} \geq \ldots \geq 0, \quad 1=f_{0}^{R} \geq f_{1}^{R} \geq f_{2}^{R} \geq \ldots \geq 0,
$$

the maximum eigenvalue in (6) is given by $\Lambda_{\max }=\Lambda_{11}^{M m}=-\alpha+f_{1}^{T} f_{1}^{R}$. Thus, the instability occurs when the global growth rate reaches its critical value $\alpha_{c}=f_{1}^{T} f_{1}^{R}$. At this instability point all nine modes with $\left(L^{u}, l^{u}\right)=(1,1)$ and $M^{u}=0, \pm 1, m^{u}=0, \pm 1$ become unstable, where we have introduced the index $u$ for the unstable modes.

\subsection{Nonlinear analysis}

In this subsection we specialize the generic order parameter equations of [1] to unit spheres. We observe that the quadratic term vanishes and derive selection rules for the appearance of cubic terms. Furthermore, we essentially simplify the calculation of the order parameter equations by taking into account the symmetry properties of the cubic terms. We show that the order parameter equations represent a potential dynamics, and determine the underlying potential.

\subsubsection{General structure of order parameter equations}

The expansion of the unstable modes (7) reads $U(\hat{t}, \hat{r})=U_{11}^{M^{u} m^{u}} Y_{1 M^{u}}^{T}(\hat{t}) Y_{1 m^{u}}^{R}(\hat{r})$ and, correspondingly, the contribution of the stable modes (8) is given by $S(\hat{t}, \hat{r})=S_{L l}^{M m} Y_{L M}^{T}(\hat{t}) Y_{l m}^{R}(\hat{r})$. Note that the latter summation is performed over all parameters $(L, l)$ except for $\left(L^{u}, l^{u}\right)=(1,1)$, i.e. from now on the parameters $(L, l)$ stand for the stable modes alone. The resulting order parameter equations (9) read

$$
\begin{aligned}
\dot{U}^{M^{u} m^{u}}= & \Lambda U^{M^{u} m^{u}}+A_{M^{u}, M^{u \prime} m^{u \prime \prime}}^{m^{u \prime} m^{u \prime \prime}} U^{M^{u \prime} m^{u \prime}} U^{M^{u \prime \prime} m^{u \prime \prime}} \\
& +B_{M^{u}, M^{u \prime} M^{u \prime \prime} M^{u \prime \prime \prime}}^{m^{u \prime} m^{u \prime \prime} m^{u \prime \prime \prime}} U^{M^{u \prime} m^{u \prime}} U^{M^{u \prime \prime} m^{u \prime \prime}} U^{M^{u \prime \prime} m^{u \prime \prime \prime} .}
\end{aligned}
$$

With the integrals (10)

$$
\begin{aligned}
I_{l, l^{(1)} l^{(2)} \ldots m^{(2)} \ldots l^{(n)}}^{m,(n)} & =\int d \Omega_{x} Y_{l m}^{*}(\hat{x}) Y_{l^{(1)} m^{(1)}}(\hat{x}) Y_{l^{(2)} m^{(2)}}(\hat{x}) \ldots Y_{l^{(n)} m^{(n)}}(\hat{x}), \\
J_{l^{(1)} l^{(2)} \ldots l^{(n)}}^{m^{(1)} m^{(2)} \ldots m^{(n)}} & =\int d \Omega_{x} Y_{l^{(1)} m^{(1)}}(\hat{x}) Y_{l^{(2)} m^{(2)}}(\hat{x}) \ldots Y_{l^{(n)} m^{(n)}}(\hat{x})
\end{aligned}
$$

the quadratic coefficients (12) read $A_{M^{u}, M^{u}{ }^{u} M^{u \prime \prime}}^{m^{u}, m^{u \prime}}=f_{1}^{T} f_{1}^{R} I_{1,1}^{M^{u}, M^{u \prime} M^{u \prime \prime}} I_{1,1}^{m^{u}, m^{u \prime} m^{u \prime \prime}}$, whereas the cubic coefficients (13) are

$B_{M^{u}, M^{u \prime} M^{u \prime \prime} M^{u \prime \prime \prime}}^{m^{u}, m^{u \prime} m^{u \prime \prime} m^{u \prime \prime \prime}}=-\frac{1}{8 \pi} f_{1}^{T} f_{1}^{R}\left(I_{1,111}^{M^{u}, M^{u \prime} M^{u \prime \prime} M^{u \prime \prime \prime}} \delta_{m^{u} m^{u \prime}} J_{11}^{m^{u \prime \prime} m^{u \prime \prime \prime}}+I_{1,111}^{m^{u}, m^{u \prime} m^{u \prime \prime} m^{u \prime \prime \prime}}\right.$ 


$$
\begin{aligned}
& \left.\times \delta_{M^{u} M^{u \prime}} J_{11}^{M^{u \prime \prime} M^{u \prime \prime \prime}}\right)+\left\{\left[f_{L}^{T} f_{l}^{R}+f_{1}^{T} f_{1}^{R}\right] I_{1,1 L}^{M^{u}, M^{u \prime} M} I_{1,1 l}^{m^{u}, m^{u \prime} m}-\frac{1}{4 \sqrt{\pi}}\left[\delta_{L 0} \delta_{M 0} \delta_{M^{u}} M^{u \prime}\right.\right. \\
& \left.\left.\times\left(1+f_{l}^{R}\right) I_{1,1}^{m^{u}, m^{u \prime} m}+\delta_{l 0} \delta_{m 0} \delta_{m^{u} m^{u \prime}}\left(1+f_{L}^{T}\right) I_{1,1}^{M^{u}, M^{u \prime} M}\right]\right\} H_{L l}^{M m, M^{u \prime \prime} m^{u \prime \prime} M^{u \prime \prime \prime} m^{u \prime \prime \prime}} .
\end{aligned}
$$

The center manifold coefficients $H_{L l}^{M m, M^{u} m^{u} M^{u \prime} m^{u \prime}}$ read

$$
\begin{aligned}
H_{L l}^{M m, M^{u} m^{u} M^{u \prime} m^{u \prime}}= & \frac{f_{1}^{T} f_{1}^{R}}{2 \Lambda-\Lambda_{L l}}\left[I_{L, 11}^{M, M^{u} M^{u \prime}} I_{l, 11}^{m, m^{u} m^{u \prime}}-\frac{1}{4 \sqrt{\pi}}\left(J_{11}^{M^{u} M^{u \prime}} I_{l, 11}^{m, m^{u \prime} m^{u \prime \prime}} \delta_{L 0}\right.\right. \\
& \left.\left.+J_{11}^{m^{u} m^{u \prime}} I_{L, 11}^{M, M^{u} M^{u \prime}} \delta_{l 0}\right)\right]
\end{aligned}
$$

\subsubsection{Integrals}

The order parameter equations contain the following integrals: $J_{11}^{m^{\prime} m^{\prime \prime}}, I_{l, 11}^{m, m^{\prime} m^{\prime \prime}}, I_{1,1 l}^{m, m^{\prime} m^{\prime \prime}}, I_{1,111}^{m, m^{\prime} m^{\prime \prime} m^{\prime \prime \prime}}$. For the first integral one easily obtains $J_{11}^{m^{\prime} m^{\prime \prime}}=(-1)^{m^{\prime}} \delta_{m^{\prime},-m^{\prime \prime}}$. Integrals over three and four spherical harmonics can be calculated using the Wigner-Eckart-theorem,

$$
I_{l, l^{\prime} l^{\prime \prime}}^{m, m^{\prime} m^{\prime \prime}}=\sqrt{\frac{\left(2 l^{\prime}+1\right)\left(2 l^{\prime \prime}+1\right)}{4 \pi(2 l+1)}} C\left(l^{\prime}, 0, l^{\prime \prime}, 0 \mid l, 0\right) C\left(l^{\prime}, m^{\prime}, l^{\prime \prime}, m^{\prime \prime} \mid l, m\right),
$$

whence for $l^{\prime}=l^{\prime \prime}=1$ it follows

$$
I_{l, 11}^{m, m^{\prime} m^{\prime \prime}}=\frac{3}{\sqrt{4 \pi(2 l+1)}} C(1,0,1,0 \mid l, 0) C\left(1, m^{\prime}, 1, m^{\prime \prime} \mid l, m\right) .
$$

As the Clebsch-Gordan coefficients $C\left(l_{1}, 0, l_{2}, 0 \mid l_{3}, 0\right)$ vanish if the sum $l_{1}+l_{2}+l_{3}$ is odd [11], we obtain $I_{1,11}^{m, m^{\prime} m^{\prime \prime}}=0$. Thus, the quadratic contribution to the order parameter equations (100) vanishes, by analogy with Euclidean manifolds. Non-vanishing integrals (106) can only occur for $l=0$ and $l=2$. Furthermore, the integrals $I_{1,1 l}^{m, m^{\prime} m^{\prime \prime}}$ follow from $I_{1,1 l}^{m, m^{\prime} m^{\prime \prime}}=(-1)^{m^{\prime}+m^{\prime \prime}} I_{l, 11}^{-m^{\prime \prime},-m m^{\prime}}$. Integrals over four spherical harmonics can also be calculated using the Wigner-Eckart-theorem, and the result is

$$
\begin{aligned}
I_{l, l^{\prime} l^{\prime \prime} l^{\prime \prime \prime}}^{m, m^{\prime} m^{\prime \prime} m^{\prime \prime \prime}}= & \sum_{l_{3}=\left|l^{\prime \prime}-l^{\prime \prime \prime}\right|}^{l^{\prime \prime}+l^{\prime \prime \prime}} \sum_{m_{3}=-l_{3}}^{l_{3}} \sqrt{\frac{\left(2 l^{\prime \prime}+1\right)\left(2 l^{\prime \prime \prime}+1\right)}{4 \pi\left(2 l_{3}+1\right)}} C\left(l^{\prime \prime}, 0, l^{\prime \prime \prime}, 0 \mid l_{3}, 0\right) \\
& \times C\left(l^{\prime \prime}, m^{\prime \prime}, l^{\prime \prime \prime}, m^{\prime \prime \prime} \mid l_{3}, m_{3}\right) I_{l, l^{\prime} l_{3}}^{m, m^{\prime} m_{3}} .
\end{aligned}
$$

Specialyzing (107) to $l=l^{\prime}=l^{\prime \prime}=l^{\prime \prime \prime}=1$ and taking into account (105) leads to $I_{1,1}^{m, m^{\prime} m^{\prime \prime} m^{\prime \prime \prime}} \propto$ $\delta_{m^{\prime}+m^{\prime \prime}+m^{\prime \prime \prime}, m}$. Thus, we obtain the selection rule that the nonvanishing integrals $I_{1,111}^{m, m^{\prime} m^{\prime \prime} m^{\prime \prime \prime}}$ fulfill the condition $m^{\prime}+m^{\prime \prime}+m^{\prime \prime \prime}=m$.

\subsubsection{Order parameter equations}

To simplify the calculation of the cubic coefficients (103) in the order parameter equations (100), we perform some basic considerations which lead to helpful symmetry properties. To this end we start with replacing $m^{u}$ by $-m^{u}$ which yields $I_{1,1}^{m^{u}}, m^{u^{\prime}} m^{u^{\prime \prime}} m^{u^{\prime \prime \prime}}=I_{1,1}^{-m^{u}},-m^{u^{\prime}}-m^{u^{\prime \prime}}-m^{u^{\prime \prime \prime}}$. Corresponding symmetry relations can also be derived for the other terms in (103). Therefore, we conclude that the order parameter equation for $U^{-M^{u}-m^{u}}$ is obtained from that of $U^{M^{u} m^{u}}$ by negating all indices $M^{u}$ and $m^{u}$ with unchanged factors. Thus, instead of explicitly calculating nine order parameter equations, it is sufficient to restrict oneself 
determining the order parameter equations for $U^{00}, U^{10}, U^{01}$, and $U^{11}$. The remaining five order parameter equations follow instantaneously from those by applying the symmetry relations, as is further worked out in [8].

To investigate how the complex order parameter equations contribute to the one-to-one retinotopy, we transform them to real variables according to

$$
\begin{array}{r}
u_{0}=U^{00} / \sqrt{2}, u_{1}=\left(U^{11}+U^{-1-1}\right) / 2, u_{2}=i\left(U^{11}-U^{-1-1}\right) / 2, \\
u_{3}=\left(U^{1-1}+U^{-11}\right) / 2, u_{4}=i\left(U^{1-1}-U^{-11}\right) / 2, u_{5}=\left(U^{01}-U^{0-1}\right) / 2, \\
u_{6}=i\left(U^{01}+U^{0-1}\right) / 2, \quad u_{7}=\left(U^{10}-U^{-10}\right) / 2, \quad u_{8}=i\left(U^{10}+U^{-10}\right) / 2 .
\end{array}
$$

The resulting real order parameter equations turns out to follow according to

$$
\dot{u}_{i}=-\frac{\partial V\left(\left\{u_{j}\right\}\right)}{\partial u_{i}}
$$

from the potential

$$
\begin{aligned}
& V\left(\left\{u_{j}\right\}\right)=-\frac{\Lambda}{2} \sum_{j=0}^{8} u_{j}^{2}-\frac{\beta_{1}}{2} u_{0}^{4}-\bar{\beta}_{2} u_{0}^{2}\left(u_{5}^{2}+u_{6}^{2}\right)-\beta_{2} u_{0}^{2}\left(u_{7}^{2}+u_{8}^{2}\right)-\beta_{3} u_{0}^{2}\left(u_{1}^{2}+u_{2}^{2}+u_{3}^{2}+u_{4}^{2}\right) \\
& -\sqrt{2} \beta_{4} u_{0}\left(u_{1} u_{5} u_{7}+u_{2} u_{5} u_{8}+u_{2} u_{6} u_{7}+u_{4} u_{6} u_{7}-u_{1} u_{6} u_{8}-u_{3} u_{5} u_{7}-u_{4} u_{5} u_{8}-u_{3} u_{6} u_{8}\right) \\
& -\beta_{5}\left(u_{5}^{2}-u_{6}^{2}\right)\left(u_{1} u_{3}+u_{2} u_{4}\right)-\beta_{5}\left(u_{7}^{2}-u_{8}^{2}\right)\left(u_{1} u_{3}-u_{2} u_{4}\right)-2 \beta_{5} u_{7} u_{8}\left(u_{1} u_{4}+u_{2} u_{3}\right) \\
& -2 \beta_{5} u_{5} u_{6}\left(u_{2} u_{3}-u_{1} u_{4}\right)+\frac{1}{2}\left[\beta_{6}\left(u_{5}^{2}+u_{6}^{2}\right)+\bar{\beta}_{6}\left(u_{7}^{2}+u_{8}^{2}\right)\right]\left(u_{1}^{2}+u_{2}^{2}+u_{3}^{2}+u_{4}^{2}\right) \\
& -\frac{\beta_{7}}{2}\left(u_{1}^{2}+u_{2}^{2}\right)\left(u_{3}^{2}+u_{4}^{2}\right)-\beta_{3}\left(u_{5}^{2}+u_{6}^{2}\right)\left(u_{7}^{2}+u_{8}^{2}\right) \\
& -\frac{\beta_{8}}{4}\left[\left(u_{1}^{2}+u_{2}^{2}\right)^{2}+\left(u_{3}^{2}+u_{4}^{2}\right)^{2}\right]+\frac{\beta_{9}}{4}\left(u_{5}^{2}+u_{6}^{2}\right)^{2}+\frac{\bar{\beta}_{9}}{4}\left(u_{7}^{2}+u_{8}^{2}\right)^{2} .
\end{aligned}
$$

The dependence of the coefficients $\beta_{i}$ and $\bar{\beta}_{i}$ on the expansion coefficients $f_{l}$ and the control parameter $\alpha$ is found in [8]. Naturally, a complete analytical determination of all stationary states of the real order parameter equations (109) is impossible. However, we are able to demonstrate that certain stationary states admit for retinotopic modes.

\subsubsection{Special case}

To this end we consider the special case where $u_{1}, u_{2}, u_{5}, u_{6}, u_{7}, u_{8}$ vanish. Then the order parameter equations (109) with (110) for the non-vanishing amplitudes $u_{0}, u_{3}, u_{4}$ reduce to

$$
\begin{aligned}
& \dot{u}_{0}=\Lambda u_{0}+2 \beta_{1} u_{0}^{3}+2 \beta_{3}\left(u_{3}^{2}+u_{4}^{2}\right) u_{0}, \\
& \dot{u}_{3}=\Lambda u_{3}+2 \beta_{3} u_{0}^{2} u_{3}+\beta_{8}\left(u_{3}^{2}+u_{4}^{2}\right) u_{3}, \\
& \dot{u}_{4}=\Lambda u_{4}+2 \beta_{3} u_{0}^{2} u_{4}+\beta_{8}\left(u_{3}^{2}+u_{4}^{2}\right) u_{4} .
\end{aligned}
$$

Due to the relation $\dot{u}_{3} / u_{3}=\dot{u}_{4} / u_{4}$ one obtains constant phase-shift angles, i.e. it holds $u_{3} \propto u_{4}$. Therefore, the system of three coupled differential equations can be reduced to two variables. To this end we introduce the new variable

$$
\xi=\sqrt{u_{3}^{2}+u_{4}^{2}}
$$


which leads to

$$
\dot{u}_{0}=\Lambda u_{0}+2 \beta_{1} u_{0}^{3}+2 \beta_{3} \xi^{2} u_{0}, \quad \dot{\xi}=\Lambda \xi+2 \beta_{3} u_{0}^{2} \xi+\beta_{8} \xi^{3} .
$$

The stationary solution, which corresponds to a coexistence of the two modes, is given by

$$
u_{0}^{2}=-\frac{\Lambda}{2\left(\beta_{3}+\beta_{8}\right)}, \quad \xi^{2}=-\frac{\Lambda}{\beta_{3}+\beta_{8}},
$$

where we used the relation $\beta_{8}=\beta_{1}+\beta_{3}$ [8]. Demanding real amplitudes $u_{0}$, $\xi$ leads to the coexistence condition $\beta_{3}+\beta_{8}<0$. Furthermore, we require stability for this state. Therefore we consider the corresponding potential $V\left(u_{0}, \xi\right)$, which can be read off from (110) and (112):

$$
V\left(u_{0}, \xi\right)=-\frac{\Lambda}{2}\left(u_{0}^{2}+\xi^{2}\right)-\frac{\beta_{1}}{2} u_{0}^{4}-\beta_{3} u_{0}^{2} \xi^{2}-\frac{\beta_{4}}{4} \xi^{4} .
$$

Stable states correspond to a minimum of $V$, which leads to the conditions $2 \beta_{3}-\beta_{8}>0, \beta_{3}-\beta_{8}>0$. If all three inequalities are valid, both the $u_{0}$ - and the $\xi$-mode coexist. If we set $u_{4}=0$, without loss of generality, the solution reads in complex variables according to (112)

$$
U^{00}=\sqrt{-\frac{\Lambda}{\beta_{3}+\beta_{8}}}, \quad U^{1-1}=U^{-11}=-\sqrt{-\frac{\Lambda}{\beta_{3}+\beta_{8}}} .
$$

Thus, the unstable part, specified in Sect. 5.2.1, is given by

$$
U(\hat{t}, \hat{r})=\sqrt{-\frac{\Lambda}{\beta_{3}+\beta_{8}}}\left[Y_{10}^{T}(\hat{t}) Y_{10}^{R}(\hat{r})-Y_{11}^{T}(\hat{t}) Y_{1-1}^{R}(\hat{r})-Y_{1-1}^{T}(\hat{t}) Y_{11}^{R}(\hat{r})\right] .
$$

Using the Legendre addition theorem reduces (117) to

$$
U(\hat{t}, \hat{r})=\sqrt{-\frac{\Lambda}{\beta_{3}+\beta_{8}}} P_{1}(\hat{t} \cdot \hat{r})
$$

with $P_{1}(\hat{t} \cdot \hat{r})=\hat{t} \cdot \hat{r}$. Thus, the unstable part is minimal, if $\hat{t}$ and $\hat{r}$ are antiparallel, i.e. the distance of the corresponding points on the unit sphere is maximum. Decreasing the angle between $\hat{t}$ and $\hat{r}$ leads to increasing values of $U(\hat{t}, \hat{r})$, and the maximum occurs for parallel unit vectors. This justifies calling the mode (118) retinotopic.

\subsection{One-to-one retinotopy}

Now we investigate whether the generalized Häussler-von der Malsburg equations (93) describe the emergence of a perfect one-to-one retinotopy between two spheres. To this end we follow the unpublished suggestions of [12] and treat systematically the contribution of higher modes. Because the Legendre functions form a complete orthogonal system for functions defined on the interval $[-1,+1]$, their products can always be written as linear combinations of Legendre functions. This motivates that the influence of higher modes upon the connection weights, which obey the generalized Häussler-von der Malsburg equations (93), can be included by the ansatz

$$
w(\sigma)=\sum_{l=0}^{\infty}(2 l+1) Z_{l} P_{l}(\sigma)
$$

where the amplitudes $Z_{l}$ are time dependent. 


\subsubsection{Recursion relations}

Inserting (119) into the generalized Häussler-von der Malsburg equations (93) and performing the integrals over the respective unit spheres leads to

$$
\begin{aligned}
\sum_{l=0}^{\infty}(2 l+1) \dot{Z}_{l} P_{l}(\sigma)= & \alpha\left[1-\sum_{l=0}^{\infty}(2 l+1) Z_{l} P_{l}(\sigma)\right] \\
& +\sum_{l=0}^{\infty}(2 l+1) Z_{l} P_{l}(\sigma) \sum_{l^{\prime}=0}^{\infty}\left(2 l^{\prime}+1\right) Z_{l^{\prime}} f_{l^{\prime}}^{T} f_{l^{\prime}}^{R}\left[P_{l^{\prime}}(\sigma)-Z_{l^{\prime}}\right] .
\end{aligned}
$$

The products of Legendre functions occuring in (120) can be reduced to linear combinations of single Legendre functions according to the standard decomposition [10, 8.915]

$$
P_{l}(\sigma) P_{l^{\prime}}(\sigma)=\sum_{k=0}^{l} A_{l, l^{\prime}, k} P_{l+l^{\prime}-2 k}(\sigma), \quad l \leq l^{\prime}
$$

with the coefficients

$$
A_{l, l^{\prime}, k}=\frac{\left(2 l^{\prime}+2 l-4 k+1\right) a_{l^{\prime}-k} a_{k} a_{l-k}}{\left(2 l^{\prime}+2 l-2 k+1\right) a_{l+l^{\prime}-k}}, \quad a_{k}=\frac{(2 k-1) ! !}{k !} .
$$

Thus, contributions to the polynomial $P_{\hat{l}}(\sigma)$ only occur iff the relation $k=\left(l+l^{\prime}-\tilde{l}\right) / 2$ is fulfilled. Furthermore, using the orthonormality relation of the polynomials yields the following recursion relation for the amplitudes $Z_{l}$ :

$$
\begin{aligned}
(2 l+1) \dot{Z}_{l}= & \alpha\left[\delta_{l, 0}-(2 l+1) Z_{l}\right]-(2 l+1) Z_{l}\left(Z_{0}^{2}+3 f_{1}^{T} f_{1}^{R} Z_{1}^{2}\right) \\
& +\sum_{l^{\prime}=0}^{\infty}\left(2 l^{\prime}+1\right) Z_{l^{\prime}}\left[\sum_{l^{\prime \prime}=0}^{l}\left(2 l^{\prime \prime}+1\right) Z_{l^{\prime \prime}} f_{l^{\prime \prime}}^{T} f_{l^{\prime \prime}}^{R} \sum_{k=0}^{l^{\prime \prime}} A_{l^{\prime}, l^{\prime \prime}, k} \delta_{k,\left(l^{\prime}+l^{\prime \prime}-l\right) / 2}\right. \\
& \left.+\sum_{l^{\prime \prime}=l^{\prime}+1}^{\infty}\left(2 l^{\prime \prime}+1\right) Z_{l^{\prime \prime}} f_{l^{\prime \prime}}^{T} f_{l^{\prime \prime}}^{R} \sum_{k=0}^{l^{\prime}} A_{l^{\prime}, l^{\prime \prime}, k} \delta_{k,\left(l^{\prime}+l^{\prime \prime}-l\right) / 2}\right] .
\end{aligned}
$$

Note that eq. (123) cannot be solved analytically for arbitrary expansion coefficients $f_{l}^{T}, f_{l}^{R}$ of the cooperativity functions. Therefore, we restrict ourselves from now on to a special case.

\subsubsection{Special cooperativity functions}

For simplicity we assume for the cooperativity functions (97) that $f_{0}=1, f_{1} \neq 0, f_{l}=0$ for $l \neq 0, \pm 1$. With this choice the recursion relation (123) for $l=0$ reduces to

$$
\dot{Z}_{0}=-\left(\alpha+Z_{0}^{2}+3 \gamma Z_{1}^{2}\right)\left(Z_{0}-1\right),
$$

where we have used again the abbreviation $\gamma=f_{1}^{T} f_{1}^{R}$. For $l \neq 0$, by taking into account (122), we obtain

$$
\dot{Z}_{l}=-\left(\alpha+Z_{0}^{2}+3 \gamma Z_{1}^{2}\right) Z_{l}+Z_{0} Z_{l}+3 \gamma Z_{1} \frac{l Z_{l-1}+(l+1) Z_{l+1}}{2 l+1} .
$$

The long-time behavior of the system corresponds to its stationary states. They are determined by $Z_{0}=1$ from (124), whereas (125) leads to a nonlinear recursion relation for the amplitudes $Z_{l}$ with $l \neq 0$. However, by introducing the variable

$$
u=\frac{\alpha+3 \gamma Z_{1}(u)^{2}}{3 \gamma Z_{1}(u)},
$$


this nonlinear recursion relation can be formally transformed into the linear one

$$
(l+1) Z_{l+1}(u)=(2 l+1) u Z_{l}(u)+l Z_{l-1}(u), \quad l \geq 1 .
$$

Thus, determining the stationary solution of the nonlinear recursion relation (125) amounts to solving the linear recursion relation (127) for $Z_{l}(u)$ in such a way that the self-consistency condition (126) is fulfilled.

\subsubsection{Generating function}

To determine the amplitudes $Z_{l}(u)$ we calculate their generating function

$$
E(x, u)=\sum_{l=0}^{\infty} Z_{l}(u) x^{l},
$$

where we have the normalization

$$
E(0, u)=Z_{0}(u)=1 .
$$

Multiplying both sides of (127) with $x^{l}$ and summing over $l \geq 1$ leads to an inhomogeneous nonlinear partial differential equation of first order for the generating function:

$$
\left(x^{2}-2 u x+1\right) \frac{\partial E(x, u)}{\partial x}=(u-x) E(x, u)+Z_{1}(u)-u .
$$

Using the normalization condition (129) its solution is given by

$$
E(x, u)=\frac{1+\left[Z_{1}(u)-u\right] \ln \frac{\sqrt{x^{2}-2 u x+1}+x-u}{1-u}}{\sqrt{x^{2}-2 u x+1}} .
$$

\subsubsection{Decomposition}

We now determine the unknown amplitudes $Z_{l}(u)$. From the mathematical literature it is well-known that the recursion relation (127) holds both for the Legendre functions of first kind $P_{l}(u)$ and second kind $Q_{l}(u)$, respectively [10]. Thus, we expect that the generating function (131) can be represented as a linear combination of the generating functions of the Legendre functions of both first and second kind, which are given by $[10,8.921]$ and $[10,8.791 .2]$ :

$$
\begin{aligned}
& E_{P}(x, u)=\sum_{l=0}^{\infty} P_{l}(u) x^{l}=\frac{1}{\sqrt{x^{2}-2 u x+1}}, \\
& E_{Q}(x, u)=\sum_{l=0}^{\infty} Q_{l}(u) x^{l}=\frac{\ln \frac{\sqrt{x^{2}-2 u x+1}+u-x}{\sqrt{u^{2}-1}}}{\sqrt{x^{2}-2 u x+1}} .
\end{aligned}
$$

Indeed, taking into account the explicit form of the Legendre function of second kind for $l=0$ [13]

$$
Q_{0}(u)=\frac{1}{2} \ln \frac{u+1}{u-1},
$$

the generating function (131) decomposes according to

$$
E(x, u)=\left\{1+\left[Z_{1}(u)-u\right] Q_{0}(u)\right\} E_{P}(x, u)-\left[Z_{1}(u)-u\right] E_{Q}(x, u) .
$$



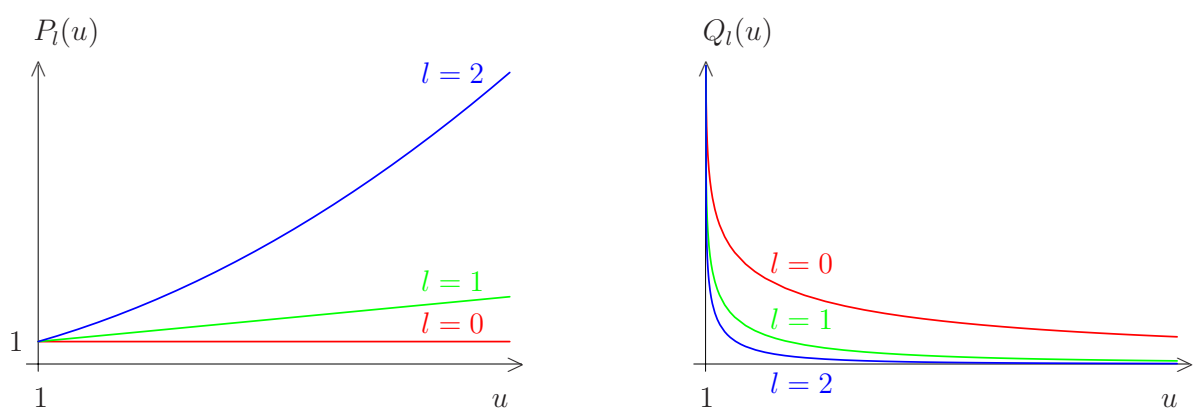

Fig. 11 (online colour at: www.ann-phys.org) The Legendre functions of first and second kind $P_{l}(u)$ and $Q_{l}(u)$ for $u>1$. We have $P_{l}(1)=1$, whereas $Q_{l}(u)$ diverges for $u \downarrow 1$. Important for the boundary condition of $Z_{l}(u)$ is the different behavior for increasing values of $l: P_{l}(u)$ diverges, whereas $Q_{l}(u)$ converges to zero.

Inserting (132), (133) and performing a comparison with (128) then yields the result

$$
Z_{l}(u)=\left\{1+\left[Z_{1}(u)-u\right] Q_{0}(u)\right\} P_{l}(u)-\left[Z_{1}(u)-u\right] Q_{l}(u) .
$$

Thus, the amplitudes $Z_{l}(u)$ turn out to be linear combinations of $P_{l}(u)$ and $Q_{l}(u)$. To fix the yet undetermined amplitude $Z_{1}(u)$ in the expansion coefficients of (136), we have to take into account the boundary condition that the sum in the ansatz (119) has to converge.

\subsubsection{Boundary condition}

Because the Legendre functions $P_{l}(\sigma)$ do not vanish with increasing $l$, we must require that $Z_{l}(u)$ vanishes in the limit $l \rightarrow \infty$. The series of Legendre functions of first kind $P_{l}(u)$ with fixed $u>1$ diverges for $l \rightarrow \infty$ according to [10, 8.917]: $P_{0}(u)<P_{1}(u)<P_{2}(u)<\ldots<P_{n}(u)<\ldots, u>1$. The Legendre functions of second kind $Q_{l}(u)$, however, converge to zero (see Fig. 11). Thus, performing the limit $l \rightarrow \infty$ in eq. (136) and using the explicit form [13] $Q_{1}(u)=u Q_{0}(u)-1$ it follows that $Z_{1}(u)$ is fixed according to $Z_{1}(u)=Q_{1}(u) / Q_{0}(u)$. With this we obtain that the result (136) finally reads

$$
Z_{l}(u)=\frac{Q_{l}(u)}{Q_{0}(u)},
$$

which is not valid only for $l \neq 0$ but also for $l=0$ due to (129).

\subsubsection{Connection weight}

Inserting (137) into (119) yields the following solution for the connection weight:

$$
w(\sigma)=\frac{1}{Q_{0}(u)} \sum_{l=0}^{\infty}(2 l+1) Q_{l}(u) P_{l}(\sigma) .
$$

Using the identity $[10,8.791 .1]$ and (134), we obtain for the connection weight

$$
w(\sigma)=\frac{2}{u-\sigma}\left(\ln \frac{u+1}{u-1}\right)^{-1} .
$$

Note that integrating (139) over the unit sphere leads to $4 \pi$, i.e. the total connection weight coincides with the measure $M$. 


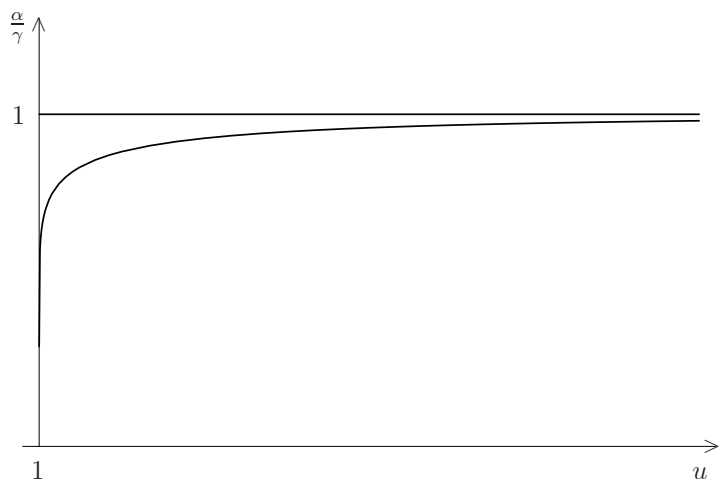

a)

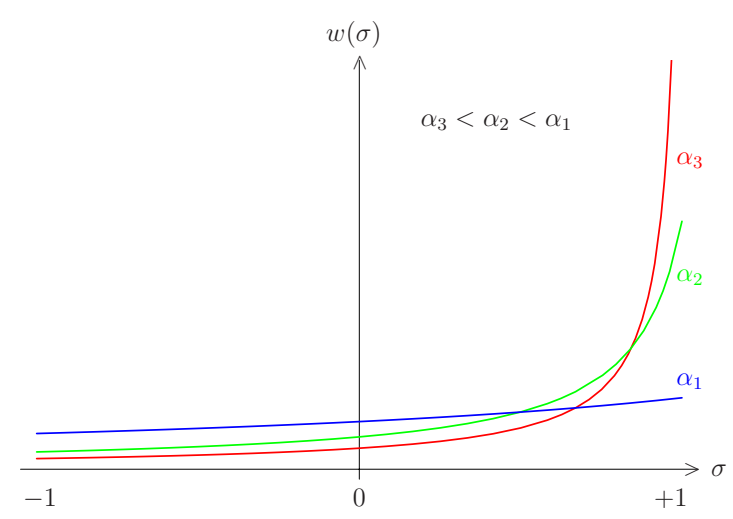

b)

Fig. 12 (online colour at: www.ann-phys.org) a) Relation (140) between the control parameter $\alpha$ and the variable $u$. b) The connection weight for different values of the control parameter $\alpha$. For decreasing values of $\alpha$ the connection weight around $\sigma=+1$ is growing. In the limiting case $\alpha \rightarrow 0$ the connection weight $w(\sigma)$ becomes Dirac's delta function (142).

On the other hand we have to take into account that the self-consistency condition (126) yields an explicit relation between the variable $u$ and the control parameter $\alpha$. Indeed, we infer from (126) and (137) the following transcendental relation between $\alpha$ and $u$

$$
\frac{\alpha}{\gamma}=-\frac{2}{3}\left(\ln \frac{u+1}{u-1}\right)^{-1}\left[2\left(\ln \frac{u+1}{u-1}\right)^{-1}-u\right],
$$

which is depicted in Fig. 12a.

\subsubsection{Limiting cases}

The limiting value of (140) for $u \rightarrow \infty$ is determined with the help of the expansion [10, 1.513]

$$
\ln \frac{1+x}{1-x}=2 \sum_{k=1}^{\infty} \frac{1}{2 k-1} x^{2 k-1}, \quad x^{2}<1,
$$

and reads $\lim _{u \rightarrow \infty} \alpha=\gamma$. Thus, we conclude that the case $u \rightarrow \infty$ corresponds to the instability point $\alpha_{c}=f_{1}^{T} f_{1}^{R}$, which was obtained from the linear stability analysis in Sect. 5.1. Correspondingly, using again (140), we observe that the connection weight (139) coincides in the limit $u \rightarrow \infty$ with a uniform distribution: $\lim _{\alpha \uparrow \alpha_{c}} w(\sigma)=1$. Another biological important special case is $u \downarrow 1$, where we obtain from the transcendental relation (140) $\lim _{u \downarrow 1} \alpha=0$. Furthermore, considering the limit $u \downarrow 1$ in (139) for $\sigma \neq u$, we obtain $w(\sigma) \rightarrow 0$. On the other hand, integrating (139) for $u \downarrow 1$ over $\sigma$ yields 2 . Therefore, we conclude that the connection weight (139) becomes in this limit Dirac's delta function:

$$
\lim _{\alpha \downarrow 0} w(\sigma)=4 \delta(\sigma-1)
$$

Thus, decreasing the control parameter $\alpha$ means that the projection between two spheres becomes sharper and sharper (see Fig. 12b). A perfect one-to-one retinotopy is achieved for $\alpha=0$ when the uniform and undifferentiated formation of new synapses onto the tectum is completely terminated. 


\section{Summary}

In this paper we have explicitly applied our generic model for the emergence of retinotopic projections between manifolds of different geometry to one- and two-dimensional Euclidean and spherical manifolds. By treating retina and tectum as strings we generalized the original approach of Häussler and von der Malsburg where both were modelled as one-dimensional discrete cell arrays. This change from discrete to continuous variables is physiologically reasonable because of the high cell density in vertebrate animals. By using a continuous instead of a discrete model we emphasized that we are not interested in the dynamics of the single cell but in the evolving global spatio-temporal patterns of the system. Furthermore, continuous variables are helpful to describe retinotopic projections between manifolds of different magnitudes as we have seen by the example of two strings of different lengths. In case of discrete cell arrays with different cell numbers it is not clear what a perfect retinotopy means, whereas in the continuous case a perfect oneto-one projection can be described without offending the bijectivity of the projections. Finally, as the onedimensional string model could only serve as a simplistic approximation to the real biological situation, we have also investigated under which conditions retinotopic projections between planar networks of neurons arise. Obviously, this increase of the spatial dimension rendered the synergetic analysis so complicated that we were only able to treat physiologically interesting special cases. While for strings retinotopy was only possible for monotonically decreasing cooperativity functions, we found that this is no longer true for planes.

Applying our generalized Häussler-von der Malsburg equations [1] to strings and to spheres, led to remarkably analogous results. Both for one-dimensional strings and for spheres we have furnished proof that our generalized Häussler-von der Malsburg equations describe, indeed, the emergence of a perfect one-to-one retinotopy. Furthermore, we have shown in both cases that the underlying order parameter equations follow from a potential dynamics and do not contain quadratic terms. However, in contrast to strings, spherical manifolds represent a more adequate description for retina and tectum. Therefore, the spherical case represents an essential progress in the understanding of the ontogenetic development of neural connections between retina and tectum.

\section{References}

[1] M. Güßmann, A. Pelster and G. Wunner, Ann. Phys. (Leipzig) 16, 379 (2007) [this issue].

[2] G. J. Goodhill and L. J. Richards, Trends Neurosci. 22, 529 (1999).

[3] A.F. Häussler and C. v. d. Malsburg, J. Theoret. Neurobiol. 2, 47 (1983).

[4] H. Haken, Synergetics - An Introduction, third edition (Springer, Berlin, 1983).

[5] H. Haken, Advanced Synergetics (Springer, Berlin, 1983).

[6] M. Güßmann, A. Pelster, and G. Wunner, Self-Organized Development of Retinotopic Projections; in: Proceedings of the 5. Workshop Dynamic Perception, Tübingen, Germany, November 18-19, 2004, edited by U. J. Illg, H. H. Bülthoff and H.A. Mallot (Akademische Verlagsgesellschaft, Berlin, 2004), p. 239.

[7] H. Kleinert, Path Integrals in Quantum Mechanics, Statistics, Polymer Physics and Financial Markets, fourth edition (World Scientific, Singapore, 2006).

[8] M. Güßmann, Self-Organization between Manifolds of Euclidean and non-Euclidean Geometry by Cooperation and Competition, Ph.D. Thesis (in german), Universität Stuttgart (2006); internet: www. itp1.uni-stuttgart.de/publikationen/guessmann_doktor_2006.pdf.

[9] W. Wischert, A. Wunderlin, A. Pelster, M. Olivier, and J. Groslambert, Phys. Rev. E 49, 203 (1994).

[10] I. S. Gradshteyn and I. M. Ryzhik, Table of Integrals, Series, and Products, fourth edition (Academic Press, New York, 1965).

[11] V. Heine, Group Theory in Quantum Mechanics (Dover, New York, 1993).

[12] W. Wagner and C. v. d. Malsburg, private communication.

[13] G. B. Arfken and H. J. Weber, Mathematical Methods for Physicists, fifth edition (Academic Press, London, 2001). 